\title{
Possible uses of path integration in animal navigation
}

\author{
ROBERT BIEGLER \\ University of Edinburgh, Edinburgh, Scotland
}

\begin{abstract}
Path integration, in its simplest form, keeps track of movement from a starting point and so makes it possible to return to this point. Path integration can also be used to build a metric spatial representation of the environment, if given a suitable readout mechanism that can store and recall the coordinates of any one of multiple locations. A simple averaging process can make this representation as accurate as desired, given enough visits to the locations stored in the representation. There are more than these two ways of using path integration in navigation. They can be classified systematically according to the following three criteria: Is there one point at which coordinates can be reset to correct errors, or several? Is there one possible goal, or several? Is there one path integrator, or several? I describe the resulting eight methods of using path integration and compare their characteristics with the available experimental evidence. The classification offers a theoretical framework for further research.
\end{abstract}

Methods of navigation can be broadly divided into those that use location-based information, which specifies (with varying precision) where an animal is by reference to specific and identifiable features of the environment, and those that use movement-based or location-independent information. Location-independent information encompasses internal, self-generated motion cues (such as efference copies of motor commands, kinesthetic cues, and inertial cues from the vestibular system, collectively called idiothetic cues; H. Mittelstaedt \& M. L. Mittelstaedt, 1973), external motion-based cues (such as optic flow, doppler effect, and air or water speed), and finally, compass cues (such as the geomagnetic field and celestial cues or landmarks that are far enough away that their bearings stay approximately constant). Location-independent information can be used to record movement from a starting point. This record may take the form of a movement sequence, the direction back to the starting point or direction and distance. Path integration is the computation, based on location-independent cues, of distance and direction or, sometimes, direction only from the current location to the starting point or vice versa.

Animals in a variety of taxonomic groups, from arthropods to mammals and birds, have been found to use path

This work was supported by the Wellcome Trust. This manuscript is partly based on a $\mathrm{PhD}$ thesis written under the supervision of R. G. M. Morris. I am grateful for his teaching and support. I also thank him and the following for critical comments on earlier versions of this manuscript: S. Benhamou, P. Boulcott, S. E. Dye, D. J. Foster, K. M. Gothard, A. S. Griffin, S. D. Healy, J. Henderson, A. McGregor, H. Mittelstaedt, O. Trullier, R. J. Willson, and several anonymous reviewers. Correspondence concerning this article should be addressed to R. Biegler, Centre for Neuroscience, University of Edinburgh, Crichton Street, Edinburgh EH8 9LE, Scotland (e-mail: r.biegler@ed.ac.uk). After September 1, 2000, address correspondence to Department of Psychology, Norwegian University of Technology and Science (NTNU), N-7491 Trondheim, Norway (e-mail: robert.biegler@svt.ntnu.no). integration (arthropods: Beugnon \& Campan, 1989; Hoffmann, 1984; H. Mittelstaedt, 1985; Müller \& Wehner, 1988; Ronacher \& Wehner, 1995; Schmidt, Collett, Dillier, \& Wehner, 1992; von Frisch, 1967; Wehner \& Räber, 1979; Wehner \& Wehner, 1990; Zeil, 1998; mammals: Etienne, Hurni, Maurer, \& Séguinot, 1991; Etienne, Maurer, \& Saucy, 1988; Etienne, Maurer, Saucy, \& Teroni, 1986; Etienne, Teroni, Maurer, Portenier, \& Saucy, 1985; M. L. Mittelstaedt \& Glasauer, 1991; H. Mittelstaedt \& M. L. Mittelstaedt, 1973; Séguinot, Cattet, \& Benhamou, 1998; Séguinot, Maurer, \& Etienne, 1993; birds: von Saint Paul, 1982). The use of path integration has been investigated extensively in ants of the genus Cataglyphis (Figure 1; reviewed by Wehner, Michel, \& Antonsen, 1996). Their habitat includes flat salt pan desert largely devoid of landmarks. The ants go on circuitous foraging trips, looking for insects killed by the heat. If a forager does not return to the nest within about an hour, it is likely to die itself. The ability to return directly to the nest, rather than retracing the outward path, is valuable. The nest entrance is a hole only millimeters across, often without conspicuous landmarks nearby, so the ants need accurate and reliable navigation. They use path integration to generate a direct trajectory back to the nest. Path integration appears to be their primary navigation method, being backed up only by landmarks.

If path integration can be that important, the question arises of whether Cataglyphis ants are a special case. Is it justified to treat path integration in most other cases only as a kind of back-up mechanism for occasions on which no landmarks are available or as a simple, quick, and dirty navigation method used in between the occasions on which more sophisticated but slower landmarkbased methods provide accurate information? O'Keefe (1976) has already suggested that path integration could, rather, be the basis for cognitive mapping. More recently, there has been renewed interest in that idea (Gallistel, 


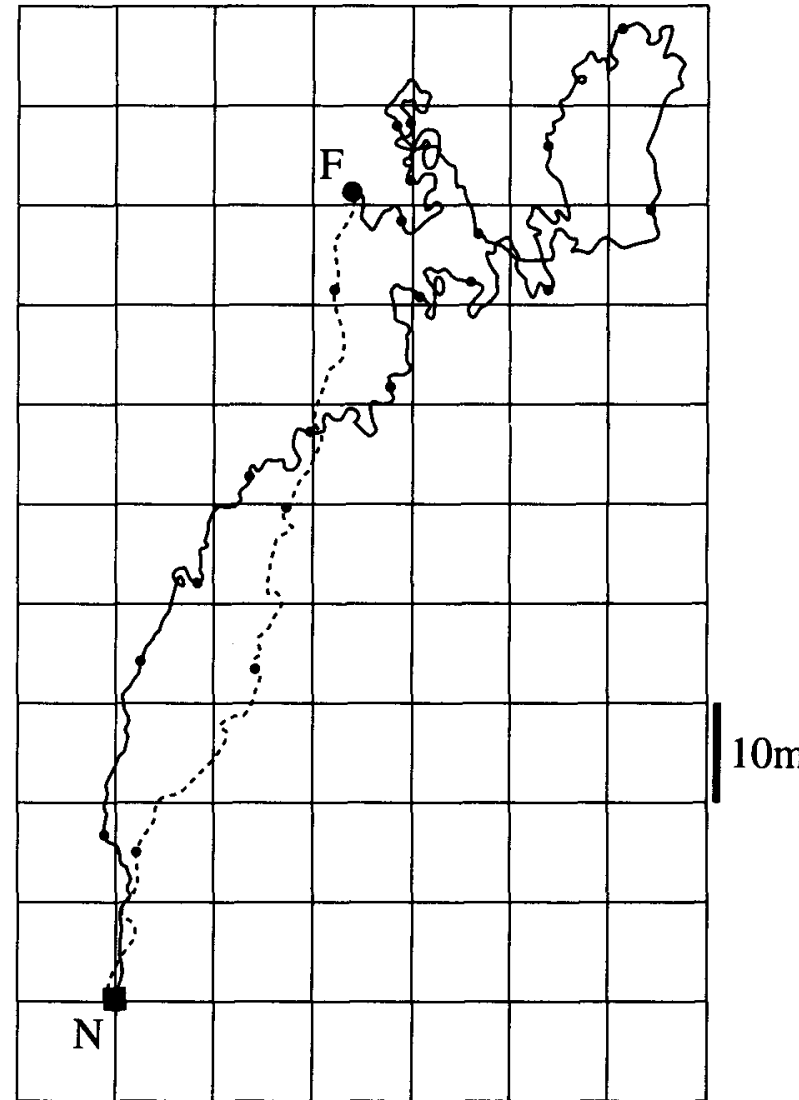

Figure 1. Foraging trip of an individual ant, Cataglyphis fortis. When the ant found food (F) after a circuitous search (solid line), it returned on a fairly straight path (stippled line) to the nest $(\mathbf{N})$. Time marks (small filled circles) are given every 60 sec. The total length of the outbound path is $354.5 \mathrm{~m}$, and the maximal distance from the nest is $113.2 \mathrm{~m}$. From "Path Integration in Desert Ants, Cataglyphis fortis," by M. Müller and R. Wehner, 1988, Proceedings of the National Academy of Sciences, 85, p. 5288. Copyright 1988 by Wehner. Reprinted with permission.

1990; Gallistel \& Cramer, 1996; McNaughton et al., 1996; Redish \& Touretzky, 1997; Samsonovich \& McNaughton, 1997; Touretzky \& Redish, 1996; Wan, Touretzky, \& Redish, 1994). Something that is missing from these treatments of path integration is a general consideration of the features a path integrator must have in order to be useful as a primary navigation system and a systematic examination of the various ways in which path integration could be used for navigation, including the limits to which it can be taken. That is what I aim to provide in this paper.

In the preliminary considerations, I show why I can exclude some issues from my argument without loss of generality. The main part of the paper is divided into two. First, I examine what features a path integrator must have if it is to be used as a major navigation system. Second, I show that these features can be added to or subtracted from models of path integration in a systematic fashion. This leads to a classification of possible path integration systems, ranging from a system that provides nothing more than a lifeline leading back to the starting point to a system that has most of the characteristics of O'Keefe and Nadel's (1978) cognitive map although it makes use of only quite rudimentary landmark-based processes for resetting.

\section{PRELIMINARY CONSIDERATIONS}

\section{Is It Necessary to Know Distance?}

Jander (1957) and Maurer (1998) both suggested, for different reasons, that animals could return to a starting point without computing the distance traveled, only taking into account the direction of travel. A path integration model that accurately represented direction to the goal (or reproduced experimentally observed biases; see Müller \& Wehner, 1988, Séguinot et al., 1993, and a review by Maurer \& Séguinot, 1995) obviously can account for all of the results from studies in which only the homing direction was measured. Although it is less obvious, such a model can also cope with findings that some animals travel for a set distance and then start searching for the goal (rats: Benhamou, 1997b; hamsters: Séguinot et al., 1993; ants: Wehner \& Srinivasan, 1981; fiddler crabs: Zeil, 1998). If an animal traveled past the location toward which its path integrator points, the indicated direction would shift very suddenly. Such a sudden shift could be used as a signal to start looking for the goal.

However, a sudden shift in the computed direction to the goal can occur only when the subject is very close to the goal, so any estimate of distance given far from the goal cannot be explained in this way. At least two species can be made to indicate their estimate of distance to a goal without traveling there. Bees communicate the distance, as well as the direction of a food source, to other foragers through their waggle dance. That distance is estimated through path integration. Humans can walk toward a goal with eyes closed, stop at a location chosen by the experimenter, and throw an object toward the goal. It is clear that at least two species, only remotely related, can represent distance in their path integrators (Esch \& Burns, 1996; Kirchner \& Braun, 1994; Thomson, 1983a, 1983b; von Frisch, 1967). Furthermore, there are cells in the rat hippocampus that show location-specific firing (place fields) that is at least partly determined by path integration (Jeffery, Donnett, Burgess, \& O'Keefe, 1997; McNaughton et al., 1996). This implies either that hippocampal place cells are part of a path integrator or that one of their inputs comes from a path integrator. If that path integrator represented only direction from a starting point, then at least under conditions in which landmark information is excluded, place fields would be wedge shaped, all meeting at one point. The finding that firing of these cells is place specific rather than bearing specific shows that the rat's path integrator also must take distance into account. Because path integration does measure distance in the only species for which conclusive ev- 
idence is available, I will, in the remaining sections of the paper, deal only with path integrators that represent both distance and direction.

\section{Path Integration Including Distance: Computations and Representations}

The principle underlying path integration is to calculate position relative to a reference point, using information about one's movements. The information available to a path integration mechanism is speed integrated over time (or distance traveled within a finite time interval, $\Delta s$ ), and direction of travel relative to some compass cue or internally generated reference direction. The computations are conventionally described in either a Cartesian or a polar coordinate system (Figure 2 ). With a Cartesian coordinate system, $\Delta s$ and the angle to the reference direction $(\lambda)$ are used to calculate the path components along $x$ - and $y$-axes. With a polar coordinate system, location is defined by the angle, $v$, between the current position, the nest, and the reference direction and by the distance, $r$, from the nest. The animal must compute the increment of radial distance, $\Delta r$, and the increment of the nest angle, $\Delta v$. Assuming that $\Delta q$, the increment of tangential distance, is small, as compared with $r$ (see also Figure 2),

$$
\begin{aligned}
& \text { Polar coordinates: } \\
& \Delta r=\Delta s \cos (\lambda-v)=\Delta s \cos d \\
& \Delta q=\Delta s \sin (\lambda-v)=\Delta s \sin d \\
& \Delta v=\Delta q / r=\Delta s \sin (\lambda-v) / r=\Delta s \sin d / r \text {. }
\end{aligned}
$$

Cartesian coordinates:

$$
\begin{aligned}
& \Delta x=\Delta s \sin \lambda \\
& \Delta y=\Delta s \cos \lambda .
\end{aligned}
$$

There is a third way of performing path integration that sidesteps computation of trigonometric functions. It is best explained by comparing use of a survey map with use of the Cartesian coordinate system printed over the map. For the coordinate system, locations are specified by a pair of numbers or some equivalent variable (e.g., the locations of peaks of neural activity in Hartmann and Wehner's, 1995, model). The $x$ and $y$ components of the last path segment are then added to the retrieved coordinates of the previous position, and the updated values are stored, for example, by writing them down. Rather than using pairs of numbers, position might also be specified by making a mark on the map. The length of the last path segment is determined by reference to a scale, its direction relative to north, or some equivalent reference. Then, using a ruler, a line can be drawn, representing the path segment, and a new mark made at its end, representing the new position. At no point is it necessary to split the path segment into two components and calculate their lengths by multiplication with a trigonometric function. Only the end result of the computation is the same. Each of these types of representation has been used in models of path integration (Hartmann \& Wehner, 1995; McNaughton et al., 1996; H. Mittelstaedt, 1983, 1985; H. Mittelstaedt \& M. L. Mittelstaedt, 1973; Müller \& Wehner, 1988; Samsonovich \& McNaughton, 1997).

The crucial point for the purposes of this paper is that Cartesian and polar coordinate systems and the graphical updating of a map are mathematically strictly equivalent. Although it has been argued that the differences between algorithms affect accuracy when implemented in a noisy biological system (Gallistel, 1990) and that they could be
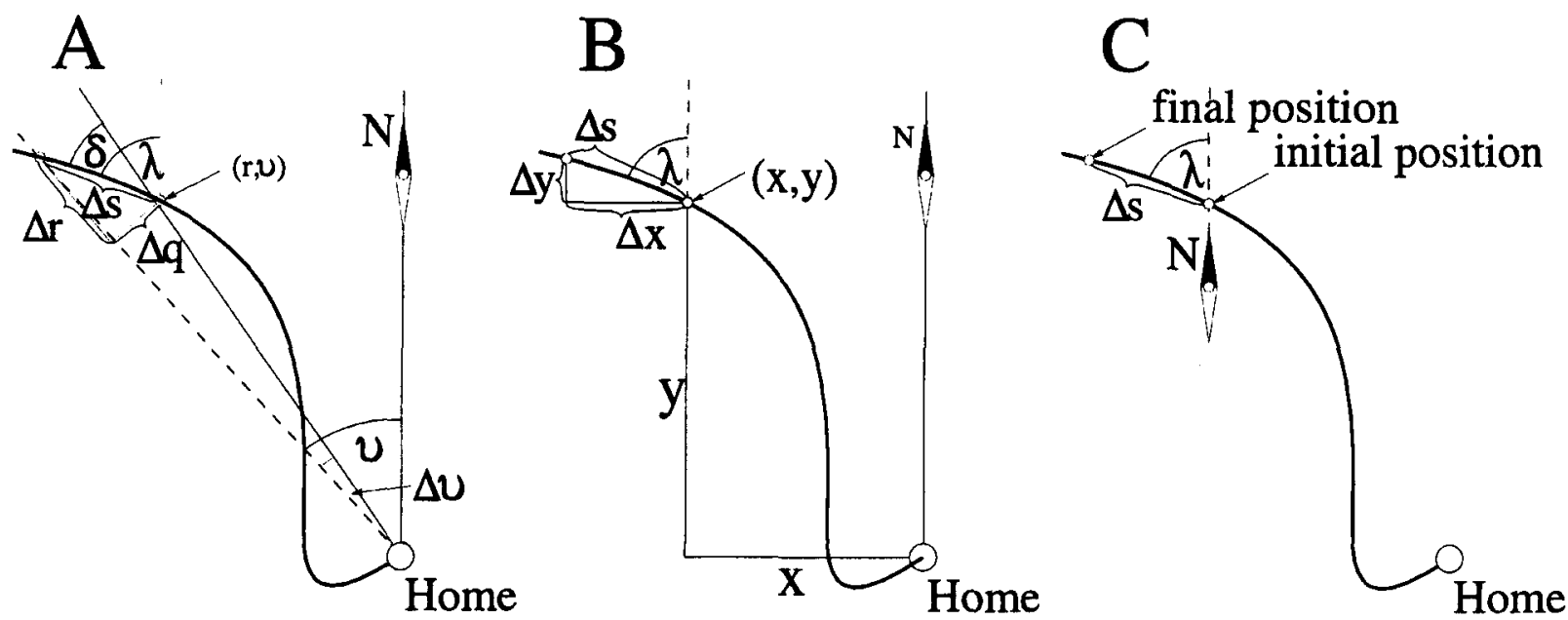

Figure 2. Information available for path integration. (A) With a polar coordinate system, the radial distance from the home $(r)$ and the angle $(v)$ between home and some reference direction describe the position $(r, v)$ of the ant. Locomotion in direction $\lambda$ by a path increment $\Delta s$ results in a new position $(r+\Delta r, v+\Delta v)$, as long as $\Delta s \ll r$. (B) With a Cartesian coordinate system, the same path increment $(\Delta s)$ and direction $(\lambda)$ are decomposed into increments, $\Delta x$ and $\Delta y$, added to the current position $(x, y)$. (C) Graphical updating of a map does not require decomposition of the path increment $\Delta s$. A marker (e.g., a coin put on a survey map or a patch of neural activation on a place cell map) is moved from an initial position by distance $\Delta s$ in the appropriate direction into the final position. Calculation of trigonometric functions is implicit rather than explicit, but the isomorphism of algebra and geometry guarantees that the result is the same. 
relevant when one is trying to account for systematic errors in path integration (Hartmann \& Wehner, 1995; Müller \& Wehner, 1988), this is not relevant for the purposes of my argument. Therefore, I will not make any such distinction.

Likewise, it is irrelevant for the purposes of this paper whether a representation is assumed to be egocentric (the animal's position in the representation is stationary, and everything else moves) or exocentric (the animal moves, and the represented environment is stationary; for a discussion of this topic, see Benhamou, 1997a). For any exocentric representation, it is possible to develop an equivalent egocentric representation. For the sake of simplicity, from here on, representations are always described as exocentric.

\section{NECESSARY FEATURES OF PATH INTEGRATION}

For a path integrator to be useful in navigation, it is not sufficient that it represents the spatial relationship between an animal's current location and some point of interest. Two more things are needed: First, because random errors accumulate over time, the animal must have a way of determining its position by reference to location-based information (fixing a position) so as to reset the path integrator to more accurate coordinates. Second, there must be a readout mechanism that can lead the animal to its goal. I will discuss readout in more detail later.

\section{Resetting of the Path Integrator: \\ Characteristics and Experimental Evidence}

Resetting has three characteristic features. All have been observed experimentally. First, if there is a discrepancy between the estimated position and the position fix, resetting revises the estimated position so as to reduce or eliminate the discrepancy. Such a discrepancy or deviation may be caused by accumulated errors in path integration, mistakes in identifying landmarks, errors in the position fix, or movement of landmarks. Gothard, Skaggs, and McNaughton (1996b) simultaneously recorded from several hippocampal place cells while a rat shuttled back and forth between a startbox and a goalbox. They found resetting of the hippocampal place field representation when they created a discrepancy between location-based and location-independent information by reducing the distance between the start- and the goalboxes, as compared with the distance used during initial training. If the distance was reduced a little, the place field representation initially reflected distance from the starting point; then it raced through the place fields corresponding to places in the central part of the runway until it reflected distance from the destination. If the distance between the start- and the goalboxes was reduced more, the place field representation skipped over the fields corresponding to the central part of the runway. Srinivasan, Zhang, and Bidwell (1997) investigated distance estimation by path integration in bees flying along a tunnel. When they shifted a landmark that the bees passed in the tunnel well before reaching the goal, the bees' search shifted accordingly, consistent with resetting of the path integrator at the landmark.

Second, after resetting, the coordinates in the path integrator should reflect the animal's position more accurately than before. I will argue that it cannot be guaranteed that resetting will improve accuracy in every instance and that specific conditions must be met even to ensure that resetting improves accuracy on average. Nevertheless, improving accuracy is the whole purpose of resetting, and accuracy can be measured. If an animal is repeatedly observed while it is searching for a goal and if, sometimes, it is given an opportunity to reset path integration near the goal, then, after resetting, the peak of its search distribution should, on average, be centered more accurately on the goal. The bees' search for food in Srinivasan et al.'s (1997) tunnel was more accurate, as measured by reduced scatter of the peaks of the search time distribution of each visit, if the bees passed a landmark earlier on in their flight through the tunnel. Griffin and Etienne (1998) found that hamsters that were passively rotated in darkness could home more accurately if the light was switched on briefly before they started the return journey.

A third characteristic of resetting is that if an animal can assess the uncertainty (the magnitude of potential error) in its estimated position based on path integration, then, after fixing its position relative to location-based cues and resetting the path integrator, its estimate of the uncertainty should decrease. Accordingly, when a goal is searched for, not only should the peak of the search distribution be centered more accurately on the goal after resetting, but the search distribution should also be narrower. This is exactly what Srinivasan et al. (1997) found when testing bees' estimation of distance by path integration.

It is functionally important to make a distinction between, on the one hand, the deviation of the estimated position from the actual position of the animal and, on the other hand, the uncertainty of the estimated position. The deviation can only be determined and eliminated when a position fix is taken. The uncertainty is a function of distance traveled and the presence of other sources of noise. Therefore, uncertainty can be estimated in the absence of a position fix.

There is at least suggestive evidence that animals do make such a distinction. Under some conditions, resetting may not occur - for example, if the deviation of locationindependent from location-based information is large (Etienne, Teroni, Hurni, \& Portenier, 1990). The reason may be that landmarks are identified partly by reference to the estimated position and orientation given by the path integrator. If the deviation is too large, landmark identification based on other information may be rejected as too inconsistent with the estimated position, in which case there would be no position fix to revise the estimated position. Effectively, the position fix would be rejected because the deviation exceeds the uncertainty of the estimated position.

A different experimental approach supports this interpretation. Rats were trained to find food at a specific dis- 
tance and direction from a landmark in an open-field arena. In the first of four tests, the rats ignored a $90^{\circ}$ counterclockwise rotation of visual and acoustic directional cues and searched in the same direction as that searched during training. Before the second test, the rats were disoriented by passive rotations on their way to the starting point, and directional cues were rotated $90^{\circ}$ clockwise. Under these conditions, the rats' direction of search relative to the landmark rotated with the directional cues. When tested in the absence of directional cues and when not disoriented, the rats searched in the same direction as that searched during training. The presence of uncontrolled directional cues could not account for this behavior, since the rats had no preferred search direction in the absence of the directional cues and when disoriented. It appears that, in the first test, a $90^{\circ}$ deviation of orientation based on vestibular cues from visual and acoustic directional cues was larger than the rats' uncertainty and did not lead to resetting, whereas the disorientation procedure in the second test increased uncertainty and, therefore, the same $90^{\circ}$ deviation from the training direction did lead to resetting (Biegler \& Morris, 1996).

\section{Matching Position Fixes to Path Integration}

It is a common assumption that a position fix provides more accurate information than does the estimated position given by a path integrator, with all its accumulated random errors. This is usually true in the special case of human navigation when accurate maps and charts are available. However, in more general terms, specific conditions must be met if position fixes are to be more accurate than path integration.

Estimations of distance through path integration and by reference to landmarks both follow Weber's law: Errors are proportional to distance (Cheng, 1990; Cheng, Srinivasan, \& Zhang, 1999; Kamil \& Jones, 1997; Srinivasan et al., 1997). If the error from path integration is a larger proportion of distance than is the error from the use of landmarks, the position fix will be, on the average, more accurate than the estimated position. On the other hand, if the likely error in distance estimated by path integration is, for example, $8 \%$, and the error in determining distance relative to a landmark is $10 \%$, then if the animal walks in a straight line from the landmark, path integration will be more accurate than a landmark-based position fix. The animal should not use a position fix to reset its path integrator. But if the path is circuitous enough to be at least $25 \%$ longer than the straight-line distance from the landmark, estimating distance from that landmark will provide more accurate information.

Of course, the animal may also obtain an accurate fix when it comes close to another landmark, but then two questions arise. How can the animal know the coordinate of the landmark or of the place from which it sees the landmark? And how can it know these coordinates with sufficient accuracy that a position fix is likely to improve on the animal's estimated position as given by path integration?

\section{Obtaining Landmark Coordinates for Resetting}

Regarding the first question (whether it is necessary to know distance), landmarks or places must be registered in a representation with the same metric and scale as and congruent with the representation used by the path integrator. If the same representation were to be used for path integration and as a map of the environment, this correspondence would be guaranteed, but separate representations are possible in principle.

At this point, it is necessary to make a distinction between animals capable or incapable of piloting. Piloting is the process of determining one's own position relative to a location of interest by first determining position relative to landmarks with known spatial relationships to the relevant location and then inferring one's own spatial relationship to that location by vector addition. In the context of resetting a path integrator, the location of interest would be the point at which path integration was initiated. An animal capable of piloting can fix its position at any place at which it can determine its distance and bearing relative to at least one identified landmark. The number and density of resetting points is effectively infinite, even if the number of known landmarks is finite.

Piloting could also be used to create the representation that is later used to provide position fixes. In principle, the animal could sit at any arbitrary location, determine the distance and bearing of each landmark, and infer the spatial relationships between them. For reasons of accuracy (see below), the animal should move between landmarks, but it is not necessary to visit them all. It is not clear whether any species has separate metric representations of space-one based on landmarks and one on path integration - and if so, how these two representations would be matched up.

Alternatively, assume that all an animal can do with landmarks is to recognize a place, $P_{1}$, perhaps by identifying a smell or a texture or by matching the currently perceived visual scene to a panorama stored in memory. It has no metric, landmark-based representation that could provide resetting coordinates. In order to obtain these, the animal must store in memory the coordinates given by the path integrator when it reaches a place that is to be used as a resetting point. It then can fix its position and reset path integration only at these specific resetting points for which it has coordinates. Because the coordinates of the resetting points are given by the path integrator in the first place, there is no need to match the scale, metric, and orientation of two different representations.

\section{How Can Resetting Be Made Accurate?}

Regarding the question of accuracy, in the case of an animal capable of piloting, there are three possibilities. If piloting is far more accurate than path integration, there is no problem. The animal can create an accurate reference frame that it can use to obtain a position fix (once it has either matched up its two representations of space or, if it has only one representation, calibrated its odometer to the scale used when piloting). 
On the other hand, if there is a significant error in the use of landmarks, random errors must first be removed by averaging. Each time the animal determines its spatial relationship to one or more of the landmarks and infers their spatial relationships to each other, the result will be different. If the process is repeated often enough and the results averaged, the representation will converge to an accurate state. It is advantageous to move around while carrying out this averaging process, because error is proportional to distance from a landmark (Cheng, 1990; Kamil \& Jones, 1997), and therefore, accuracy in estimating the spatial relationship among landmarks will improve as the animal comes closer. Once a sufficiently accurate representation of landmark locations is available, resetting is possible at any place at which a familiar landmark is visible and close enough to allow a position fix with less error than is likely to have accumulated in the path integrator.

The third possibility is to use the only process of creating an accurate frame of reference that is available to an animal not capable of piloting: averaging out random errors. As before, I will assume not only that an animal is incapable of piloting, but also that all it can do with landmarks is to recognize a place, $P_{1}$, and distinguish it from other places, $P_{2}$ to $P_{n}$ (for reasons of efficiency, it will also need a way of finding a place $P_{n}$ quickly when it is near; image matching, as described by Cartwright \& Collett, 1982, 1983, would be one candidate mechanism). Whenever the animal arrives at $P_{1}$, it stores the current coordinates given by the path integrator and averages them with coordinates already in memory. It does the same for other places, $P_{2}$ to $P_{n}$. Given sufficient sampling, the coordinates of these places will accurately reflect the spatial relationships between them, even without any capacity for piloting. Resetting will then be possible at each of these specific points but nowhere else (the number and density of resetting points is finite).

It is, therefore, possible not just to get coordinates for resetting from a path integrator, but also to make these coordinates accurate without relying on piloting or any landmark-based process more sophisticated than the ability to recognize a location (and, perhaps, image matching, in order to find a resetting point quickly when near to it).

\section{Possible Implementation of the Averaging Process}

Although there has not been, to my knowledge, any attempt yet to incorporate an averaging process into models of path integration, this can be done quite easily. I will take as an example a model by Samsonovich and McNaughton (1997). They proposed that hippocampal place cells are part of a path integrator. Location is represented by the ensemble of active place cells (Figure 3 ). This ensemble can be thought of as a compact packet of activity that moves over a two-dimensional chart as the animal moves through the environment (for a detailed explanation of both how the activity packet moves and why this topographical ordering of place cells is permissible for illustration, even though neighboring hippocampal place cells do not have neighboring place fields, see Samsonovich \& McNaughton, 1997).

A resetting point is established by linking units responsive to a place-specific visual scene to simultaneously active place cells (the visual units are hypothetical; no anatomical correlate has yet been identified). If the same scene is encountered again later (recognition of the scene constituting a position fix), activation of place cells through these connections will create an activity packet at the same coordinates in the place cell representation. Inhibitory interconnections ensure that there can be only one stable activity packet in a chart; therefore, if the packet representing the animal's estimated position is at different coordinates, the packet will either move to the location given by the position fix or fade while a new packet is forced into existence.

The crucial point is that if the links between visual units and place cells are permanently established at asymptotic strength on a single occasion, any error made by the path integrator when the resetting point is established will be retained and will be reinstated every time the animal resets path integration at this point. Such a fixation of an initial error would be avoided if several visits are needed to establish permanent connections of asymptotic strength. If errors in path integration over successive visits are random, only links from visual units to place cells at the correct coordinates will be strengthened during most or all of the visits. This way of averaging coordinates over successive visits to a place could be implemented in one or both of two ways. The learning rate might be low enough that connections could only grow to asymptotic strength over several visits. Or connections might decay unless activated repeatedly and, thus, consolidated. The path integration model of Samsonovich and McNaughton (1997) can incorporate averaging by use of appropriate learning rules, without special computational machinery. This implementation of an averaging process is not unique to the model of Samsonovich and McNaughton but can be applied to any model with a distributed place code.

The same argument applies to the storage of accurate goal coordinates. Burgess, Recce, and O'Keefe (1994) and Burgess and O'Keefe (1995) proposed a model of hippocampal function that involves readout of goal coordinates by goal cells (see below). Goal cells must be linked to place cells in order to specify the goal coordinates, in much the same manner as Samsonovich and McNaughton's (1997) visual units are linked to place cells to allow resetting. A low learning rate or the use of connections that decay unless consolidated by repeated activation would guarantee that goal coordinates approach accurate values over time. The use of goal cells is entirely compatible with a model that assumes hippocampal place cells are primarily driven by path integration, so a single model can incorporate averaging of both resetting coordinates and goal coordinates. Again, the principle can be applied not only to Burgess et al.'s model, but to any 

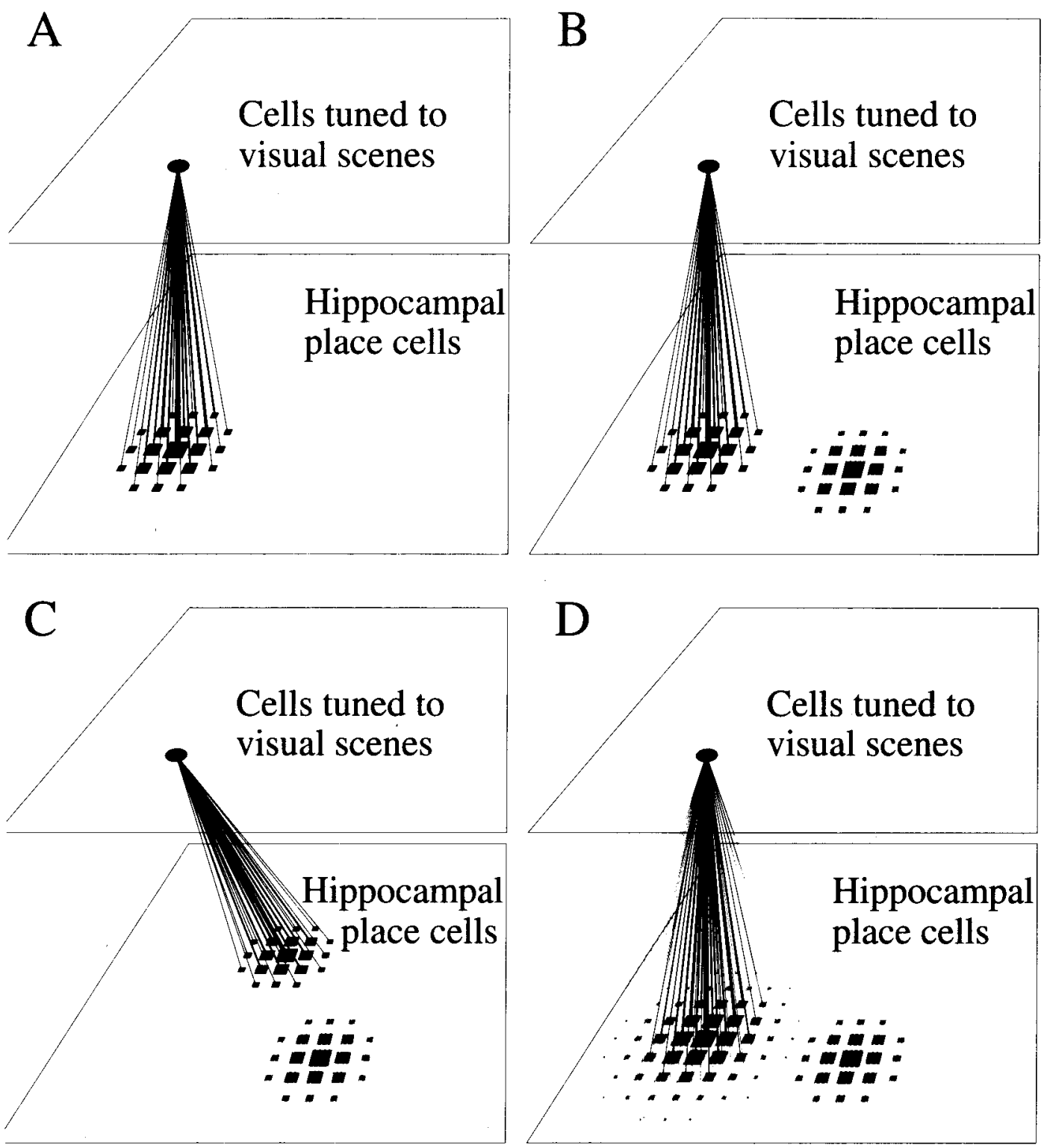

Figure 3. Resetting of path integration in the model of Samsonovich and McNaughton (1997) and removal of errors by averaging. For simplicity, place cells are arranged as if cells with adjacent place fields were also anatomically adjacent. Likewise, a unit tuned to recognize a visual scene is shown as if units tuned to neighboring scenes were anatomically adjacent. Place cells are represented by squares, whose size is proportional to the firing rate of the cell. (A) Connections from a cell or group of cells tuned to a specific visual scene to simultaneously active place cells are potentiated. The more active a place cell is, the stronger the connection from the visual unit(s) to the place cell (shown here as the thickness of the connecting lines). In this example, visual unit(s) and place cells match up exactly. (B) The animal finds itself at the point at which it stored a visual scene, but owing to an error in path integration, the activity packet representing the animal's estimated position (gray squares on the lower right) is some distance from where it should be. Activation of the visual unit will excite the correct set of place cells through the connections established earlier. Inhibitory connections between place cells ensure that there can be only one stable activity packet in the place cell representation. When an activity packet is forced into existence at the coordinate to which visual units are linked (black squares), the original packet (gray squares) either moves there or fades as a new one is created. (C) If there was an error in path integration when the animal first linked a visual scene to place cells and if the learning rate is high enough that learning effectively occurs in a single trial, any error made on that occasion will be retained. Recognition of the visual scene would reset path integration to the erroneous coordinates given when the animal established the link. (D) If the learning rate is low enough that connections from the visual unit(s) to place cells are established over a number of visits to the resetting point, each with a randomly varying error in path integration, connections from the visual unit(s) to place cells will cover a larger area but are likely to be centered far more accurately on the correct coordinates. In Samsonovich and McNaughton's model, the broader spread of the connections would not affect the size of the activity packet, which is determined by inhibitory connections. Even if the activity packet established initially is large (as shown by the gray squares on the lower left of diagram (D), it would shrink to normal size (black squares). 
model that reads out goal location through cells connected to a distributed place code.

\section{How Important Is Averaging, and What Does It Imply?}

Resetting is needed to remove accumulated errors from the position estimate. That is only possible if the resetting coordinates are accurate. No model of path integration can be considered complete unless it can explain how animals may learn coordinates for resetting and how they ensure that these coordinates are, on average, more accurate than those currently provided by the path integrator. Unless it can be shown that a species is capable of piloting and that piloting is inherently more accurate than path integration, this can only be achieved by some sort of averaging process. If there is more than one goal, averaging is also necessary in order to ensure that the goal coordinates accurately reflect the goal locations. Only if there is just one goal, which is also the only place at which the path integrator is ever reset, is averaging not necessary.

One implication of the existence of an averaging process is that there should be no asymptotic, one-trial spatial learning in an unfamiliar environment. When an animal arrives at a novel place, it is likely that its path integrator will have some error. The animal's estimate of the coordinates of this new goal location will become more accurate over successive visits. So even if the animal's memory of the goal coordinates is strong enough after one visit to find the goal again, accuracy would improve over repeated visits, and so learning would not be asymptotic after a single trial. In a thoroughly familiar environment, an animal would have established accurate resetting points by averaging out errors. Then, when it finds a new goal location, it is likely that its path integrator will estimate position accurately the first time round, and so there is no benefit (other than possibly making the memory long-lasting) from later visits to the same place. This claim is consistent with experimental evidence. Rats can benefit from a single trial in a water maze they have not seen before but they still benefit from further trials (Keith \& McVety, 1988; Whishaw, 1991). Asymptotic one-trial spatial learning has been found only when rats were repeatedly trained to find new goal locations in a familiar water maze (Steele \& Morris, 1999).

If cognitive mapping is based on path integration, a low learning rate or a need for consolidation would be advantageous, or even necessary if there is no other way to implement averaging. This contradicts O'Keefe and Nadel's (1978) claim that learning in a cognitive map is an all-or-nothing event, with an entry into a map either there or not there.

Averaging may also be one functional reason for having place fields of different sizes. Jung, Wiener, and McNaughton (1994) found that place cells in the ventral hippocampus have larger place fields than do cells in the dorsal hippocampus. The intuitively obvious interpretation that these are representations with different scale and resolution is wrong. Zhang and Sejnowski (1999) showed that resolution is independent of place field size in a twodimensional representation. A look at Figure 3 suggests another possible function. For illustrative purposes, in that figure the navigational error is much larger than the radius of a place field. It is clear, then, that many visits to a location would be needed so that the connections from place fields near the correct coordinates would be strengthened repeatedly. A given desired accuracy should be achieved more quickly if place fields were larger. A quantitative analysis would be necessary to see whether there is an optimum size of place field for a navigational error of a given magnitude. If so, there should be a range of place field sizes, because the magnitude of navigational error will not be the same on different occasions.

\section{Some Ways of Reading Out the Goal Location}

Four quite different readout mechanisms will serve to illustrate the possible range of features. The intuitively most obvious way of reading a path integrator is to extract the distance and direction of the goal from the representation, turn toward the goal, and travel until the distance is zero. Hartmann and Wehner (1995) described a neural implementation of just such a readout mechanism. Their model represents location in a polar coordinate system, directly in terms of distance and direction. In its homing mode, the model compares the animal's heading with its direction from the goal and generates turn commands until the animal is headed toward the goal. Then it gives a run command until distance has been reduced to zero. So in its present form, Hartmann and Wehner's path integrator can only create motor commands to return to the zero coordinate, and therefore, it could only be used to find this one location.

Burgess et al. (1994; see also Burgess and O'Keefe, 1995) suggested that goal cells might read the distance and direction of a goal from the hippocampal place field map in a rather different manner (because the firing of hippocampal place cells is at least partly controlled by path integration, anything that reads information from the hippocampal place field map can be considered a readout mechanism for path integration). When at a goal location (and only then), goal cells are connected to all place cells active at the time. Once these connections are established, the firing rate of a goal cell is, in the simplest case, the weighted sum of the firing rates of all the place cells it is connected to. There is a feature of hippocampal physiology that makes it possible to identify place fields that are not centered directly at the animal's current location but lie immediately in front. By turning on the spot and connecting goal cells only to place cells in front, an animal could create a set of goal cells whose firing fields surround the goal (Figure 4). So if there was one goal cell to the north of the goal, one to the east, one to the west, and one to the south, their relative firing rates would indicate the direction of the goal, whereas the sum of firing rates would be a function of distance. Such groups of goal cells could be established at any arbitrary location 


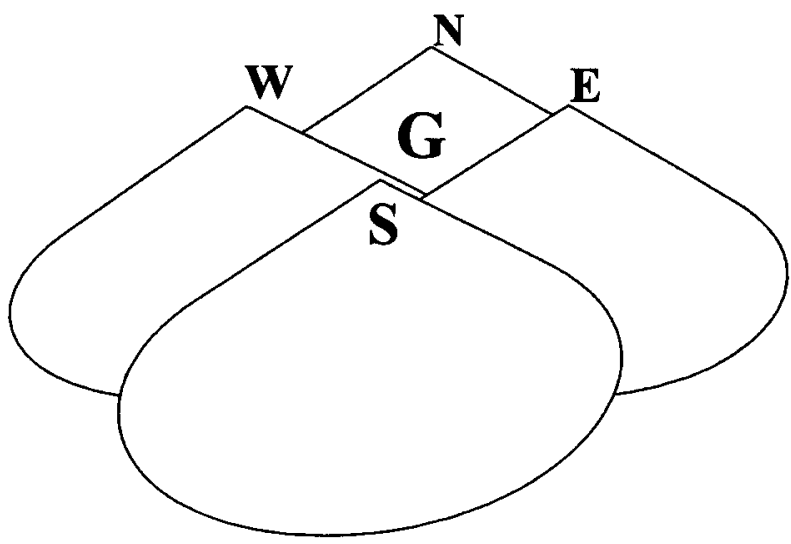

Figure 4. Firing fields of goal cells in the model of Burgess, Rece, and O'Keefe (1994). At the goal, the animal looks in several directions. Each time it selects the place cells with fields that lie ahead and connects those to a goal cell. In this example, four goal cells offset from the goal (G) in four different directions $(\mathrm{N}, \mathrm{E}, \mathrm{S}$, and $\mathrm{W})$. The relative firing rates of the goal cells specify direction to the goal; the sum of their firing provides a measure of distance. From "A Model of Hippocampal Function,” by N. Burgess, M. Recce, and J. O'Keefe, 1994, Neural Networks, 7, p. 1070. Copyright 1994 by Elsevier Science. Reprinted with permission.

the animal has visited. As long as there is a way of selectively activating one such group of goal cells, the animal can read out the location of any goal. However, it would need additional computational machinery in order to select the nearest of several goals (it must store each distance in memory and then compare them), to calculate distance between goals, or to plan a short path between several destinations.

It is not necessary to extract distance and direction from the representation, as was demonstrated by the example of H. Mittelstaedt's (1983, 1985; see also H. Mittelstaedt \& M. L. Mittelstaedt, 1973) model of path integration. This model represents location as $x$ - and $y$ coordinates in a Cartesian coordinate system. It uses the animal's heading and the difference between the animal's current coordinates and the goal coordinates, not to compute the direction and beeline distance to a goal, but to find out whether the goal is in front of or behind the animal and whether it is to the right or to the left of the current heading. The model then directly generates separate turn and run commands. Any point in space is characterized by a combination of these commands. The animal moves and turns until the turn commands cancel, then moves in a straight line to the goal, where the run commands cancel (Figure 5). Using this mechanism, an animal could travel to any goal, but estimating the distance to a goal, choosing among several destinations based on distance, or planning a path between several destinations would require additional computational mechanisms.

Reid and Staddon (1998) proposed a readout mechanism that does not explicitly deal with any spatial parameters at all. It does not use coordinates, distances, or directions in its computations. Rather, an expectation value is attributed to each location. Initially, only the goal locations have an expectation value above zero (Figure 6A). This expectation then spreads to neighboring locations in a manner analogous to diffusion (Figure 6B). The animal chooses a path by comparing, at each step, the loca-
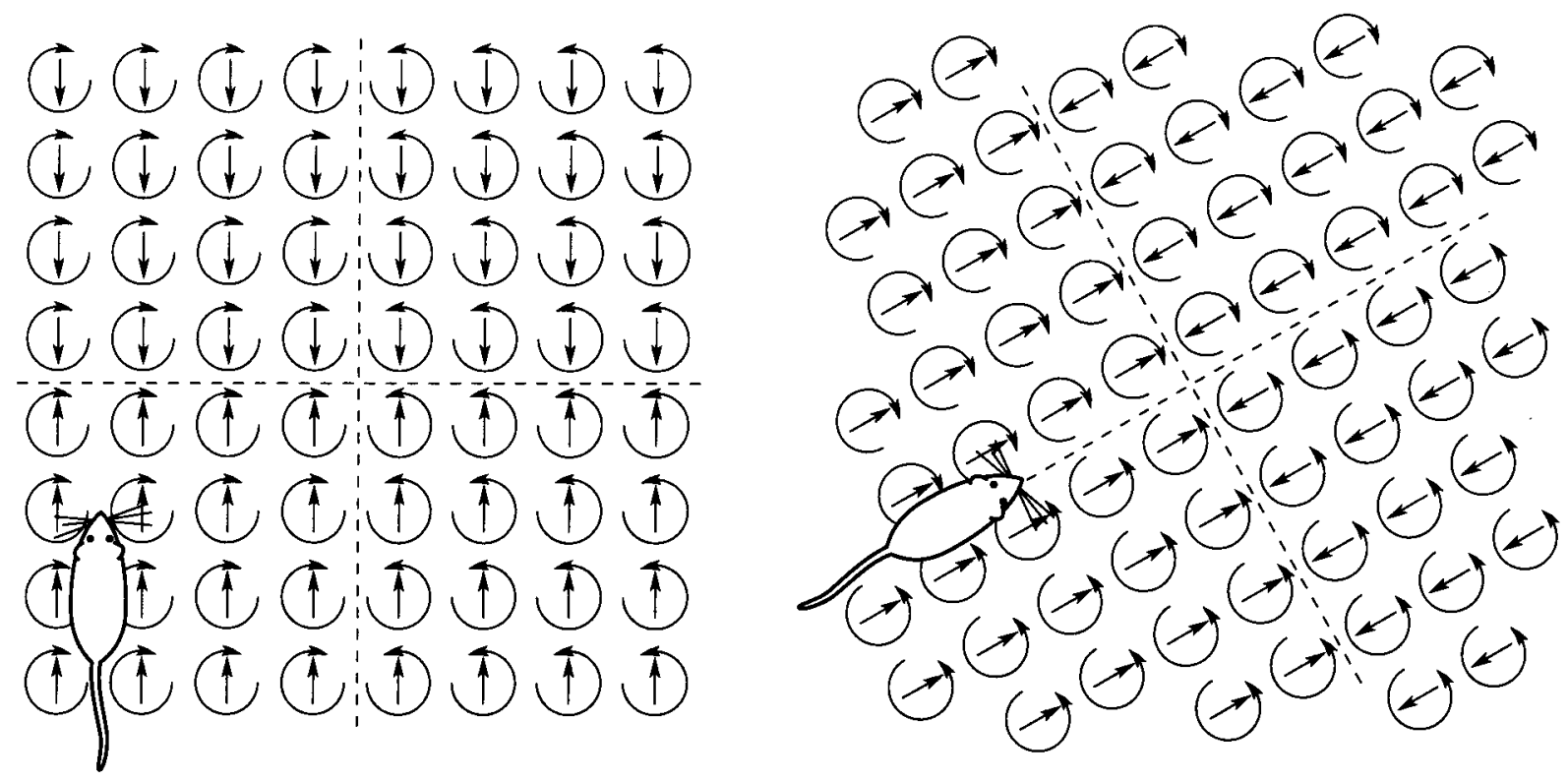

Figure 5. Run and turn commands (shown as straight and curved arrows) in H. Mittelstaedt's (1985) model of path integration. For a given heading of the animal, each point in space is characterized by a combination of run and turn commands. The broken lines show where commands cancel. The intersection of these lines is always at the goal. Their orientation depends on the animal's heading. In the location shown on the left, the animal must turn right and move forward to approach the goal. Once it has turned far enough (right diagram), right and left turn commands cancel, and it moves straight toward the goal. 

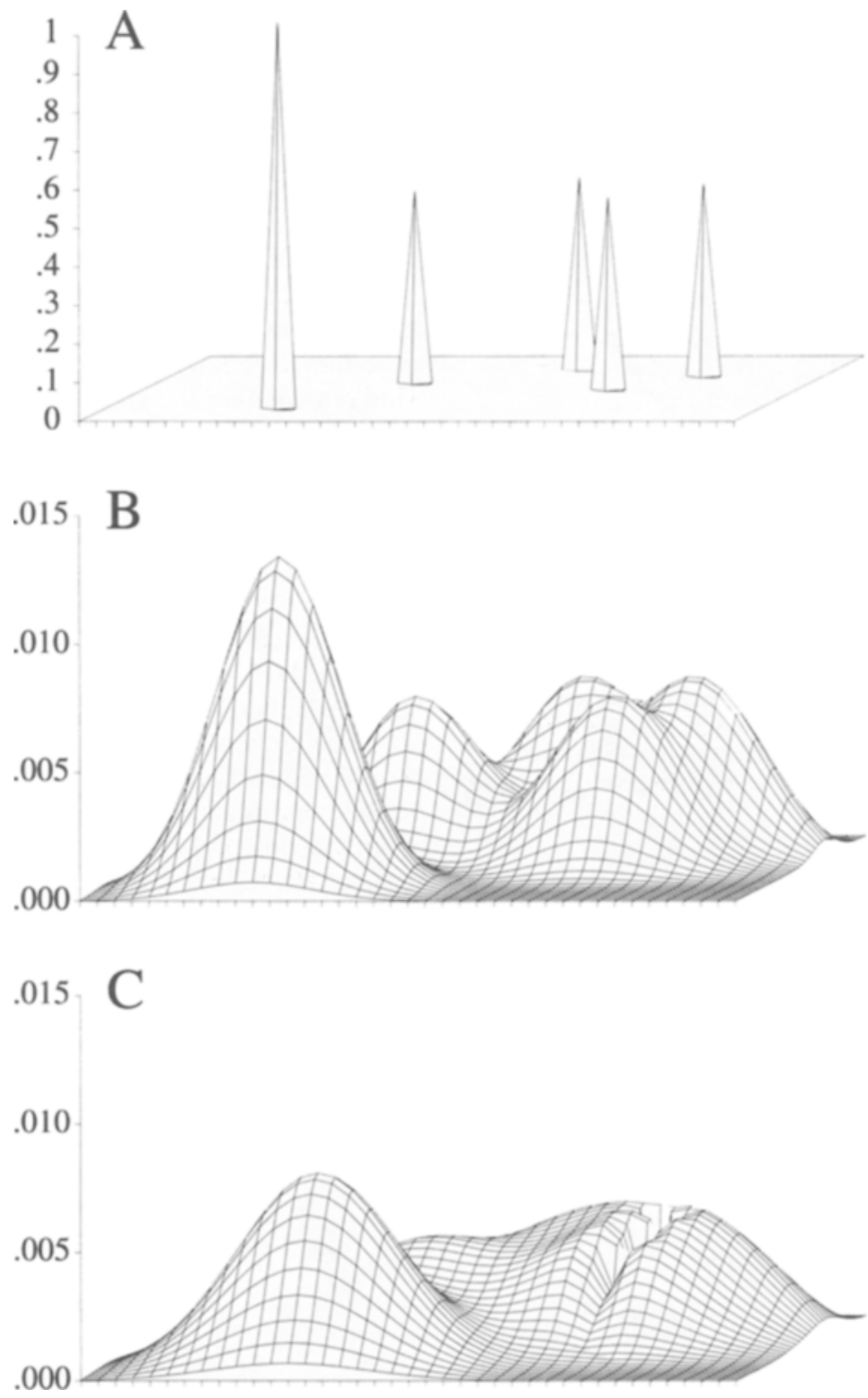

Figure 6. Plots of expectation values for all locations in an area for Reid and Staddon's (1998) model of the readout for a map. (A) Five locations are given an initial expectation value that is proportional to the size of the reward found there. (B) This expectation spreads to neighboring locations by a process analogous to diffusion. Note different scale. (C) A model animal has started searching for reward in the lower right corner of the diagram. At each point, it chooses to travel to the neighboring location with the highest expectation value. If there is no reward or when it has consumed the reward, it sets expectation at its current location to zero. This pushes down the expectation surface and prevents the animal from backtracking immediately to an already depleted reward. As the animal moves away, expectation diffuses back in from neighboring locations. In Reid and Staddon's intentionally simple version of this model, there is only a single layer of units, representing both expectation (shown here) and location. If the model were adapted so as to be a neurophysiological model, there would need to be three layers, each connected to both of the others in an ordered fashion: one layer of place cells representing location, one layer of cells implementing the spread of expectation (shown here), and a layer of goal cells representing the locations at which expectation is "injected" into the system. Furthermore, there would need to be a mechanism for comparing expectation values at locations adjacent to the current one. Reid and Staddon also coded location in the activation of just one unit at a time, but adaptation of the model to a distributed place code, as found in the hippocampus, would be straightforward. 
tions immediately adjacent to its own and selecting the one with the highest expectation value. Whenever it fails to find reward or when it has consumed the reward it has found, it sets the expectation value at its current location to zero (Figure 6C). The dynamic nature of the expectation surface prevents the animal from getting permanently caught in a local maximum of expectation. The computation of a path even between multiple goals is implicit in the generation of expectation values. This readout mechanism can provide a solution to the traveling salesman problem of finding a short path between multiple destinations (see Bureš, Burešová, \& Nerad, 1992, and Cramer \& Gallistel, 1997, for studies in rats and monkeys, respectively) without ever retrieving any coordinates or explicitly computing distances.

These four examples illustrate that there is a wide variety of ways to read out spatial information. For the purposes of this paper, the crucial point is that some readout mechanisms can only be used to find one specific goal, whereas others can deal with multiple goals. It must be noted, however, that no readout mechanism has yet been identified in any species. They must all be considered hypothetical.

\section{PATH INTEGRATION INCLUDING DISTANCE: ITS USE IN NAVIGATION}

If there are multiple resetting points, it is necessary to retrieve the correct coordinates and to have a mechanism that can reset to any arbitrary coordinate (because resetting coordinates cannot be known in advance). Also, if a path integrator is to be used to find any of several goals, there must be ways of storing and retrieving the corresponding coordinates, as well as selecting a goal. The whole system would be simpler if it had to deal with only one goal and/or one resetting point. Finally, there may be only one path integrator, or there may be several, working independently. That leads to eight possible combinations. Not all of these will be examined in detail, since some relevant data can be used to reject more than one possible scheme, at least for the species for which data are available.

\section{Single Path Integrator With Single Resetting Point and Goal}

Here, it is assumed that path integration may be initiated and reset only at a home location. Then, the path integrator will always only have information about the spatial relationship between home and the current location and so can only be used to return home, not to find any other goal (Figure 7). A physical analogue of such a path integrator would be a measuring tape tied to a weight that is put down at the goal. Resetting the path integrator is equivalent to moving the weight if it has been disturbed. To do that, one must be at the goal (assume that the weight is too heavy to move by pulling at the tape, or the analogy breaks down). If a polar or Cartesian coordinate system is used, the origin would usually be set at the location the animal wants to find again. As an example, the model of
Hartmann and Wehner (1995) makes exactly this assumption, and the neural implementation of the readout mechanism would have to be substantially modified in order for the animal to be able to find a path to a destination other than the origin.

This simple version of a path integrator can be rejected for bees, rats, hamsters, and humans. Bees can approach an arbitrary goal specified by another forager's waggle dance, and they use path integration for doing so (von Frisch, 1967). Kirchner and Braun (1994) also found that bees use path integration to determine the location of a food source they signal in a waggle dance. They captured bees at a feeding station $10 \mathrm{~m}$ south of the hive and let them fly in a wind tunnel, in which a moving pattern created optic flow. After varying flight durations, the bees emerged $1 \mathrm{~m}$ east of the first feeding station and were fed. On their return, they had an equivalent flight in the wind tunnel. Those bees that danced reported the new feeding station as due east, at distances up to several hundred meters, depending on the duration of the flight in the wind tunnel. Therefore, they must have used path integration to determine the feeder location, because if they had used landmarks to identify the feeder location, they would have reported a location just east of south, at a distance of $10 \mathrm{~m}$. Furthermore, it is clear that the bees also used path integration to find the hive on the return flight. If the bees were left to fly home on their own after an outbound flight through the tunnel, they did not turn north toward the hive only $10 \mathrm{~m}$ away but flew about $200 \mathrm{~m}$ west before circling and searching for the hive, an estimated position of the hive consistent with the optic flow they had been exposed to in the tunnel. Chittka and Kunze (1995) also reported that bees used path integration to find their way back to the hive.

Furthermore, bees have to be able to reset path integration at the hive before they set out on a foraging trip, but they can also reset path integration while on their way (Srinivasan et al., 1997). Rats shuttling back and forth between a startbox and a goalbox reset their hippocampal place field representation at both ends (Gothard et al., 1996b). Hamsters can also find more than one destination by path integration (Etienne et al., 1991; Etienne et al., 1998) and can reset path integration while on a foraging trip (Griffin \& Etienne, 1998). Humans can view up to four goals in a novel environment, close their eyes, walk past the goals, turn and then walk, with eyes still closed, to any of these goals (Rieser, Guth, \& Everett, 1986). They must be able to keep track of at least four points. In a number of other experiments, human subjects also kept track of several points without visual feedback (Easton \& Sholl, 1995; Farrell \& Thomson, 1993, 1998; Loomis et al., 1993). Human noninstrumental marine navigation is based on path integration, and position fixes are obtained whenever available (Gladwin, 1970; Lewis, 1994).

\section{Single Path Integrator With Multiple \\ Resetting Points but Only One Goal}

Resetting at any arbitrary point requires the ability to replace the current vector or pair of coordinates in a path 


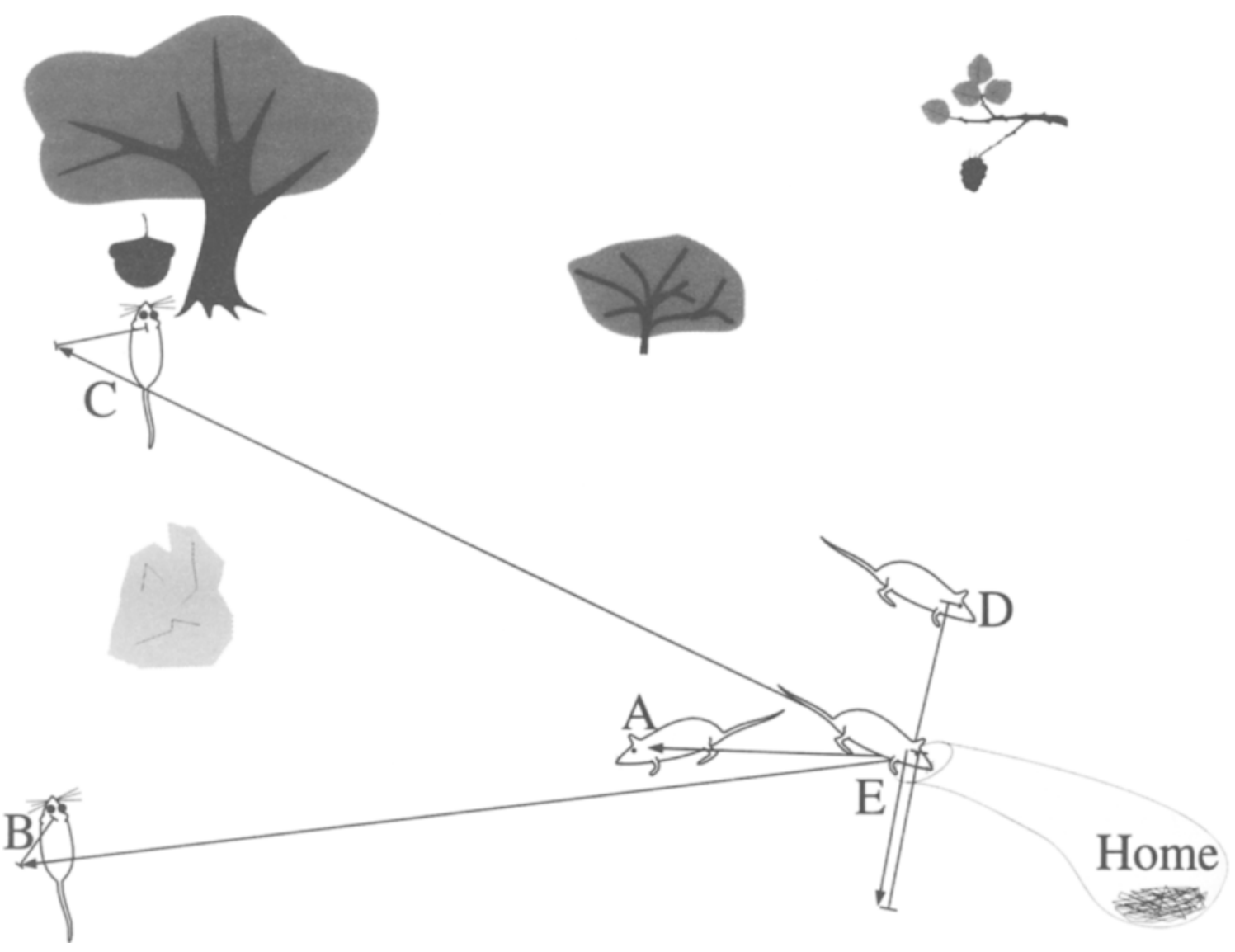

Figure 7. Path integration with resetting at and return to only one point. As an animal sets out from home, it keeps track of where it has gone from its starting point (in this case, home). This is represented here as a vector from the starting point to the animal's estimated position but could equally well be represented by a vector pointing from the animal to the estimated position of the starting point. Initially, the vector accurately reflects the spatial relationship between the starting point and the animal's current location, and so the estimated and the actual positions coincide (A). As the animal travels, errors in path integration accumulate $(B-E)$, which is represented here by an error bar showing the deviation between the animal's estimated position (at the end of the arrow) and its actual position. When the path integrator indicates that the animal should be home, it may still have some way to go (D). As the animal searches for and finally finds home, the estimated position necessarily moves farther away from home $(E)$. Before the next foraging trip, the animal must reset the vector so that its calculated starting point is home. In this simplest example, both resetting and homing are restricted to one single point. The path integrator keeps track of only one spatial relationship, that between the starting point and the animal's estimated position. The path integrator can lead the animal back only to the point at which path integration was initiated (in this case, home), with some deviation caused by accumulated errors. Because the path integrator can be reset only to a zero vector, it must be reset only when the animal has returned home. If the path integrator were reset to the zero vector while the animal was under way, it could not lead the animal home. Therefore, this way of using path integration for navigation does not allow error correction while under way.

integrator by a different, arbitrary vector. This ability has two uses. First, the new vector could be retrieved from long-term memory and used to reset the path integrator, for error correction. A physical analogy for this is to attach a measuring tape, not to a dead weight to which one has to return to carry it to a new position, but to a remotely controlled vehicle. At recognizable places, one makes a note of the distance on the tape and the direction in which it points. If, on a return to one of these points, there is a deviation, the vehicle is moved until the distance and the direction correspond to what was noted down. That is only useful if the stored vector is more accurate in relation to the goal than the current one. Accuracy could be achieved by an averaging process, as was described in the Necessary Features of Path Integration section.

This scheme still has at least one potential problem. An animal must be able to find its resetting points. Unless these occur at such a high density that the animal is guaranteed to come across resetting points quite frequently, that means the resetting points must also be goals. Although resetting and finding a goal are logically independent, they are functionally related. This scheme 


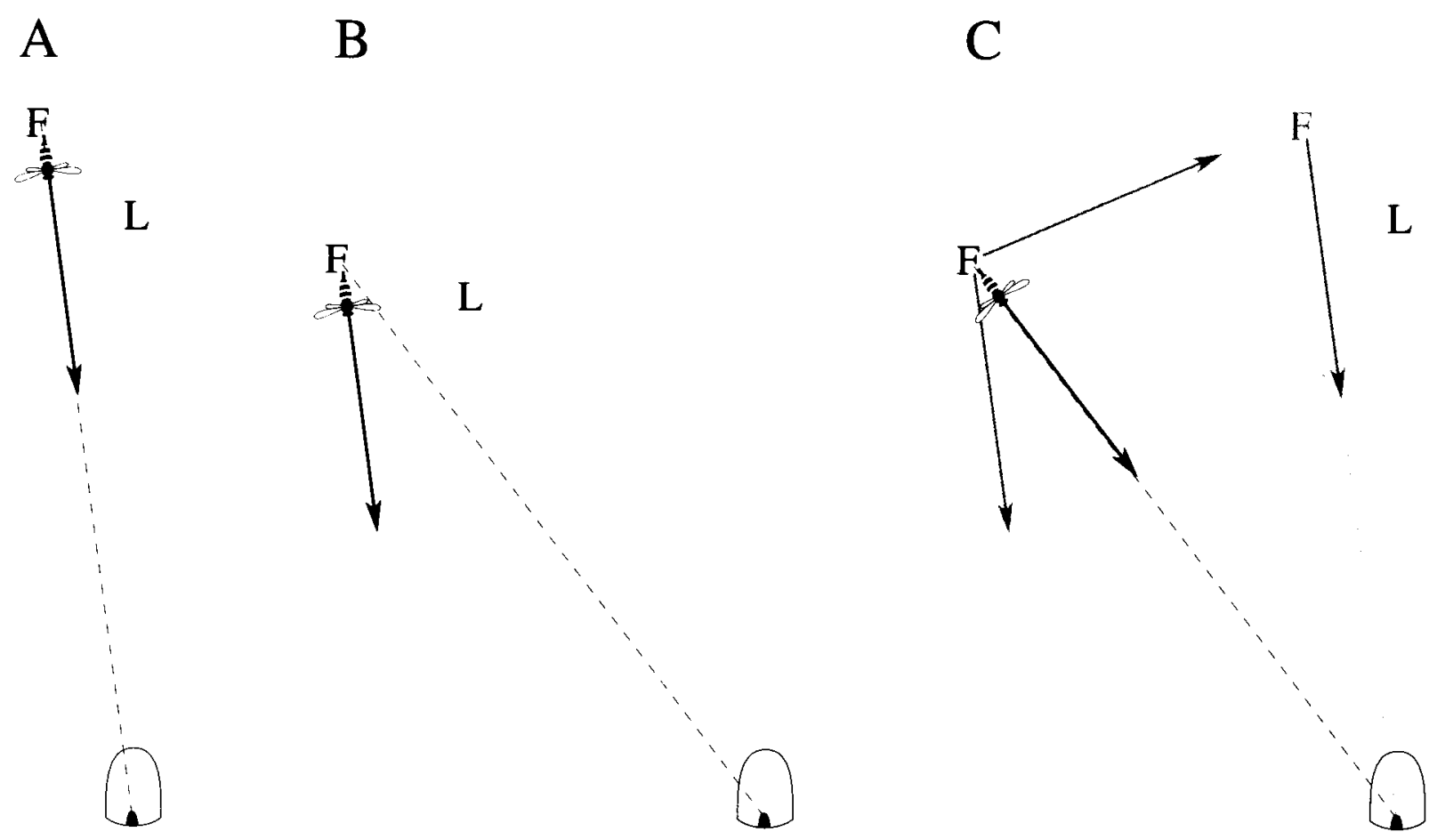

Figure 8. Resetting to move the whole frame of reference, including the goal. In the experiment of Chittka and Kunze (1995), bees flew to a feeder $(F)$ at a bearing of $352^{\circ}$ from the hive. Neither the feeder nor the landmark was visible from the hive, and the hive was not visible from the feeder. (A) During training, the bees returned directly from the feeder to the hive. (B) A change of the bearings of both the feeder and the landmark by $30^{\circ}$ simulates an error in path integration on the outbound journey. If resetting were used to redefine the goal, the bees would first need to retrieve the stored hive-feeder vector and then reset the path integrator to reverse the vector, in order to fly back to the hive. Consistent with this scheme, the bees departed from the feeder on the same course as that during training. (C) Moving only the feeder before any bee arrives there, but not the landmark, simulates depletion of the original food source and finding a different food source elsewhere. If resetting were used to redefine the goal, the bees would have two choices on their first return flight: Either they fly back on the same bearing as during training, which would not lead them to the hive, or they fly first to the original feeder location (light gray arrows and letter F), which still serves as the goal location of the path integrator, retrieve the hive-feeder vector, and reset path integration so that the hive becomes the goal. In contrast to this prediction, the bees flew straight home, showing that they had kept track of the hive location and ruling out the possibility that they used resetting to redefine the goal location.

can be rejected for bees, hamsters, and humans, who have all been shown to be capable of finding more than one place by path integration (see the preceding section).

Alternatively, resetting a path integrator to an arbitrary vector could serve a different function: It could be used to redefine the single goal location. Assume that a bee has to find a food source by path integration, either because it read the location from another forager's waggle dance or because the goal, the landmarks near the goal, and intermediate landmarks are not visible from the hive, as in Chittka and Kunze's (1995) experiment. In that experiment, bees flew to a food source $175 \mathrm{~m}$ from the hive at a bearing of $352^{\circ}$ (Figure 8 ). When starting its foraging trip at the hive, a bee may store that distance and direction in memory, then reset its path integrator so that the goal is no longer at the hive but at the food source. In order to return from the food source to the hive, the bee would need to retrieve from memory the distance and direction of its intended destination and reverse that vector.

Given the assumption that the path integrator can keep track of only one spatial relationship, that between the goal and the animal's estimated position, shifting the frame of reference so that the feeder becomes the goal excludes keeping track of position relative to the hive. The only information the bee can store for later retrieval is the vector to its intended destination; it cannot predict in advance where else it might end up. Obviously, it could compute the hive location by adding the vector from its estimated position to its destination to the destinationhive vector, but then this capacity for vector addition would require explanation. The assumption here is that the animal can only reverse the vector it set out on, not compute a new vector.

In Chittka and Kunze's (1995) experiment, bees learned to return from the feeder directly to the hive. When Chittka and Kunze moved both the feeder and a nearby landmark to a point also $175 \mathrm{~m}$ from the hive but at a bearing of $322^{\circ}$, the bees set off on their return journey on the same bearing as that during training, although that bearing was wrong by $30^{\circ}$. This is consistent with the bees' resetting their apparently erroneous path integrator at the feeder, then retrieving the vector they set out on from the 
hive and reversing it. Then, Chittka and Kunze moved only the feeder, not the landmark. This manipulation simulates depletion of a foraging patch, rather than a navigation error, since the landmark is still in its old position, far from the new feeder location.

If the bees had used resetting in order to redefine the goal, they would have had two choices at the feeder: Either they could have left on the same bearing as that during training and after the shift of both landmark and feeder, or they could have flown at a bearing of $67^{\circ}$ toward their original destination, where they could have retrieved the coordinates of that destination from memory in order to reverse the vector and fly home. Instead, the bees flew from the new feeder location directly to the hive. Bees' navigational abilities cannot be accounted for by use of a single path integrator, which has multiple resetting points but can find only one goal, not even if re- setting is used to redefine the goal. However, M. Collett, T. S. Collett, and Wehner (1999) interpreted ants' behavior in displacement experiments in these terms.

\section{Multiple Independent Path Integrators, Each With Single Resetting Point and Goal}

Another possible way to keep track of several places would be to use several path integration mechanisms, as described in the Single Path Integrator With Single Resetting Point and Goal section, working in parallel and independently. An inactive path integrator may initiate path integration at some point of interest, to allow return there later. The path integrators do not interact in any way. Each keeps track of its own goal entirely independently of what is happening with the other path integrators. Knowing the coordinates given by one path integrator would not give any information about where those

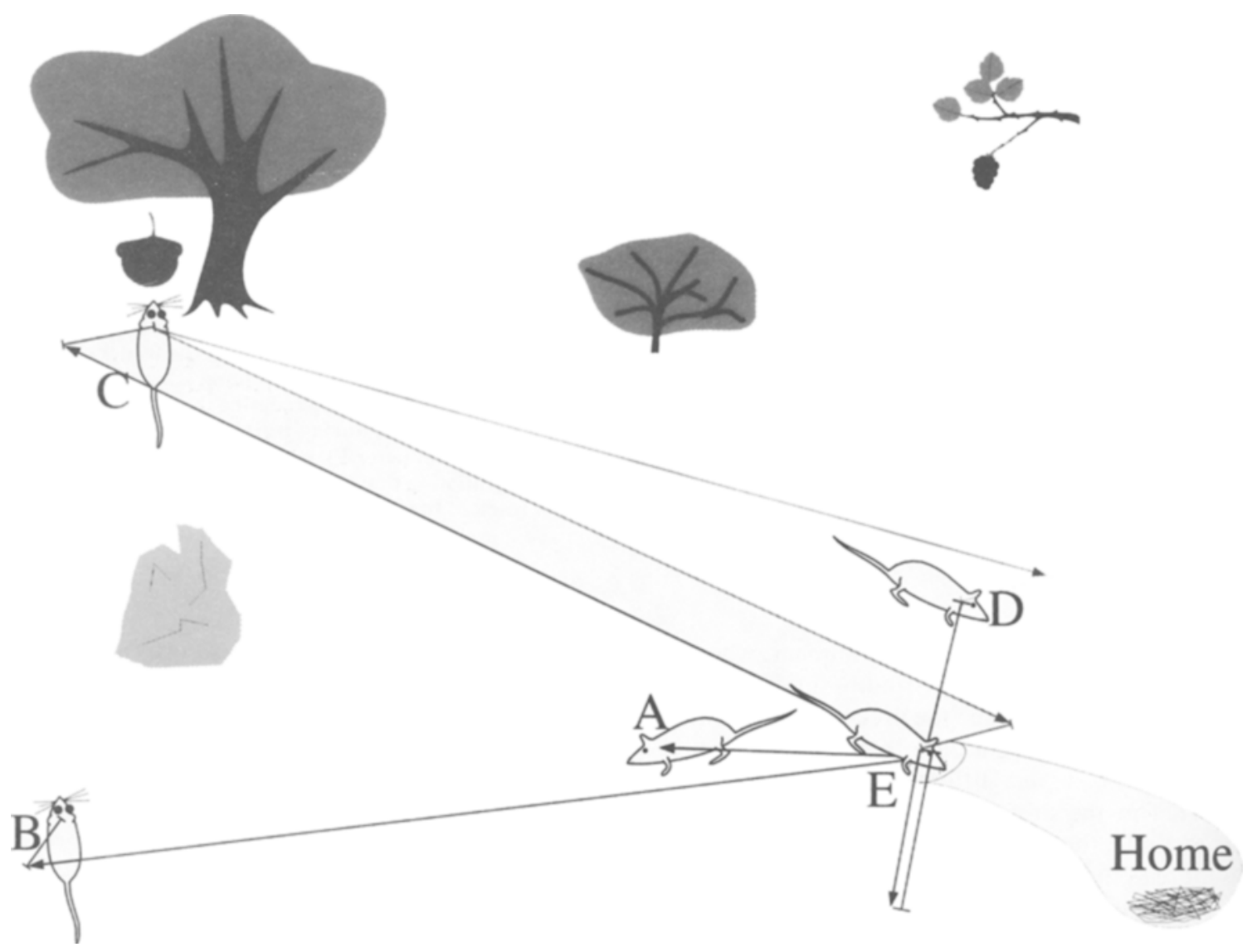

Figure 9. Multiple path integrators, each with resetting at and return to only one point. An animal goes on the same foraging trip as that in Figure 6. At $C$, it finds acorns under an oak tree. It starts up a second path integrator, keeping track of its movements from the moment it leaves the oak (represented by gray arrows). The two paths are equally affected by any errors in the estimation of distance or direction. Otherwise, they are completely independent, and each works as if the other did not exist. On arrival at $D$, the estimated position of home, the second path integrator has accumulated a somewhat different error. When the animal arrives home $(E)$ and resets the first path integrator, this does not affect the error accumulated by the second path integrator. All the animal's movements until it returns to the oak will accumulate further errors in the estimated position relative to the oak, given by the second path integrator. Only on arrival at the oak, where the second path integrator was started up, can it be reset. In turn, the resetting of the second path integrator will make no difference to any other path integrator started up somewhere else. One consequence is that, on a journey from home to the oak and back, the estimated position in the second path integrator depends on information coming in only during the return journey from the oak toward home. 
A

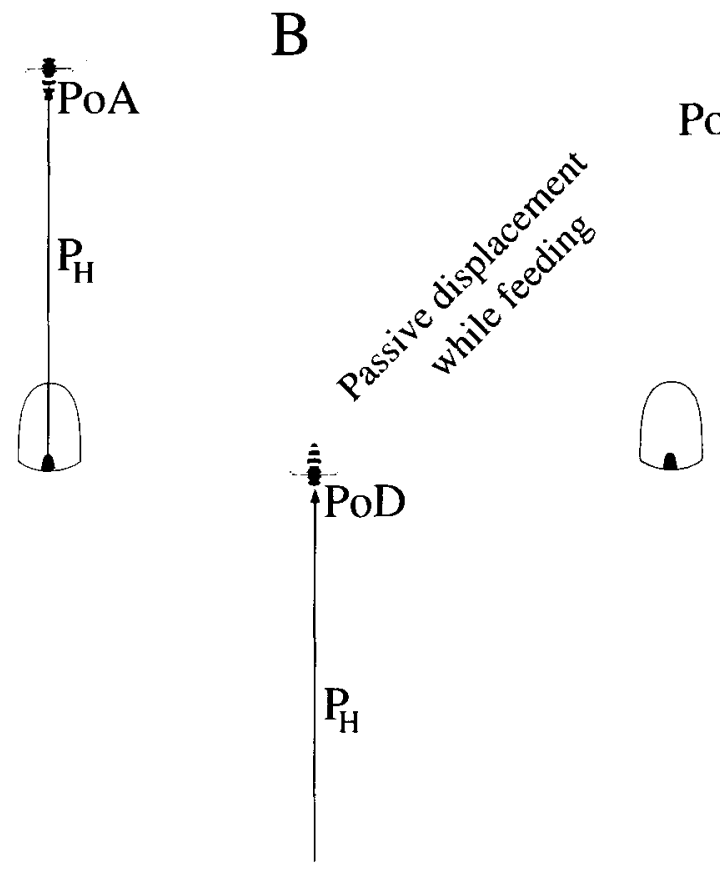

C PoA

PoA

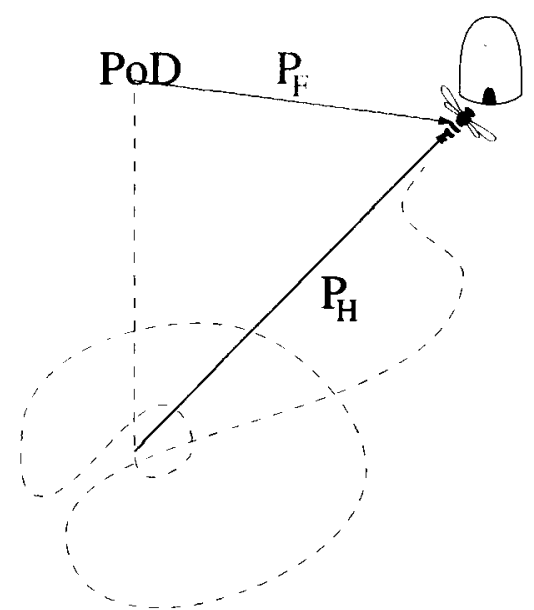

Figure 10. Summary of part of an experiment by Lindauer (1963). (A) Bees were recruited, through the waggle dance of experienced foragers, to fly to a feeder $(\mathrm{PoA}$, for point of arrival). All the experimental manipulations were carried out on the bee's first flight, when any landmarks they encountered were still unknown. If the bees used independent path integrators, one of them $\left(P_{H}\right)$ would keep track of the bee's estimated position relative to the hive. (B) As soon as a bee started feeding, the feeder was covered up and transported to another location, at the same distance from the hive, where the bee was released ( $P o D$, for point of departure). (C) Lindauer does not report the initial bearing of the return flight but only states that it was not direct. In analogy with the results of Kirchner and Braun (1994), it seems safe to assume that if the place of arrival was north of the hive, bees would initially fly south, then search for the hive (broken line). If bees use independent path integrators, they would start up a second path integrator $\left(P_{F}\right)$ when leaving the feeder, which would estimate position relative to this point of departure. On their next foraging flight, bees should, therefore, fly to their point of departure. Instead, Lindauer found that they flew to their point of arrival, suggesting that they used coordinates stored in memory when they started feeding. Bees do not use multiple independent path integrators to keep track of hive and food locations.

places are whose coordinates are defined relative to another frame of reference. Errors in estimating distance and direction would be shared between the path integrators. Random errors in computation would be independent, and the location estimates of the path integrators would drift, relative to each other (Figure 9). In the analogy used before, that would be equivalent to using several measuring tapes, each attached to a different weight. If one of the weights is disturbed, this does not affect the others.

The characteristic feature of this scheme becomes apparent when an animal travels between two points, using separate path integrators to keep track of its position relative to each point, and experiences conflicting locationindependent information on each part of the journey. Lindauer (1963) carried out just such an experiment (see Figure 10). He captured foraging bees newly recruited through the waggle dance as soon as they arrived, for the first time, at the feeder and transported them to a different location while they were feeding. If bees used one path integrator, $\mathrm{P}_{\mathrm{H}}$, to keep track of position relative the hive and another, independent path integrator, $\mathrm{P}_{\mathrm{F}}$, that they start up at the feeder, the bee's estimated position relative to the feeder, provided by $P_{F}$, can be affected only by information received on the return journey to the hive. When searching for the feeder again, the bees would fly to their place of departure, where they started their return journey to the hive, not to the place of arrival, where they started feeding and were captured.

Lindauer found that if the feeder was more than $50 \mathrm{~m}$ from the hive, 28 out of 33 bees retraced their original outbound journey, suggesting instead that, on arrival, they had stored the coordinates of the feeder relative to the hive. Srinivasan et al. (1997) also found that bees' estimate of distance depended on how far they flew into a tunnel, rather than on the distance flown when leaving the tunnel. Extended training is necessary to make bees compromise between outbound and return journey (Otto, 1959), and recent experiments by M. Collett et al. (1999) 
in ants gave similar results. In a displacement experiment, multiple path integrators of the type discussed here can only ever lead an animal back to its point of departure. If an animal returns instead to the original point of arrival, or compromises between the two, use of multiple independent path integrators, each keeping track of only one goal, cannot account for this behavior.

\section{Multiple Independent Path Integrators, Each With Multiple Resetting Points but a Single Goal}

This type of path integrator would deal with multiple goal locations in the same way as the system described in the preceding section. Lindauer's (1963) experiment provides evidence against multiple independent path integrators's dealing with one goal each, regardless of whether they each have one or several resetting points (see the preceding section for a discussion).

\section{Single Path Integrator With \\ Single Resetting Point and Multiple Goals}

Path integrators with resetting at only one point can be rejected, at least for bees, rats, hamsters, and humans (Gladwin, 1970; Gothard et al., 1996b; Griffin \& Etienne, 1998; Lewis, 1994; Srinivasan et al., 1997), as has already been discussed in the Single Path Integrator With Single Resetting Point and Goal section. The characteristic features of an ability to deal with multiple goals are, therefore, discussed in the next section.

\section{Single Path Integrator With \\ Multiple Resetting Points and Goals}

When the animal using this type of path integrator arrives at a place that is worth returning to, it stores the coordinates of that place in memory (Figure 11). It can later return to these coordinates or to any other coordinates retrieved from memory. At any one time, the path integrator would only represent the animal's estimated position relative to one location-namely, the current goal-but the animal can switch from one goal to another. Because any coordinate can be a destination, there is no need to use a different path integrator for each destination (although there may still be reasons to use separate path integrators for different areas, each of which may contain multiple destinations; see below). Effectively, each path integrator constitutes a common reference for all the goals whose coordinates are stored in memory, guaranteeing efficient information storage and internal consistency. Any random error affects the computed spatial relationships to all remembered locations equally (Figure 11 ). Likewise, resetting at a point with reliably known coordinates would correct errors in the representation of spatial relationships to all destinations.

The ability to treat any remembered place as a destination effectively constitutes a capacity for vector addition. If the stored locations are thought of as vectors to some reference point, the vector to the destination is the sum of the vector from the current location to the reference and the vector from the reference to the destination.

Benhamou (personal communication, 1998) has suggested that path integration may represent only distance and direction relative to a home location but that animals could store goal coordinates in memory. They could then retrieve the distance and direction of the goal and head straight from home toward a goal. If forced to make a detour, or if traveling from one goal location to another, they would have to match the retrieved goal coordinates to the current coordinates by a trial and error process. For example, a biased random walk (see Benhamou \& Bovet, 1992) may be used to reduce the difference between the animal's current bearing from home and the goal's remembered bearing, and the same could be done for distance. I consider this scheme to be a variant of a path integrator with multiple goals, which uses a different readout mechanism for the home location than for other locations. In either case, it is necessary to retrieve goal coordinates from memory and to replace the current set of goal coordinates with another set.

\section{Multiple Independent Path Integrators, Each With Multiple Goals and Either \\ Single or Multiple Resetting Points}

A path integrator with multiple goals can provide a complete metric representation of space. At first sight, there seems to be no need to postulate use of several such path integrators. However, the averaging process that is necessary to remove random errors from the representation takes time, and that time grows with the area covered. An animal may save time by first constructing accurate representations of small areas, only loosely linked together. Within such an area or reference frame, the animal could determine distance and direction to any point registered in the representation, but knowledge of the spatial relationships between reference frames may initially be limited to neighborhood relations. The overall representation of space would initially consist of a topological network of small, accurately represented areas, much as Poucet (1993) proposed. If multiple reference frames do speed up the construction of a spatial representation when path integration is inaccurate, animals without an external compass should benefit most, because directional errors degrade the overall accuracy of path integration more than errors in estimating distance do (Benhamou, Sauvé, \& Bovet, 1990).

From this argument, it is not clear whether or how these separately represented areas would eventually link up into an overall map of the environment, as Worden (1992) argued. It just suggests that there may be multiple frames of reference even if an animal has path integrators that can deal with multiple goals and resetting points. The existence of multiple reference frames has also been advocated, for a variety of theoretical reasons and to account for empirical data, by other authors (Davis, 1986; Gothard, Skaggs, \& McNaughton, 1996a, 1996b; 

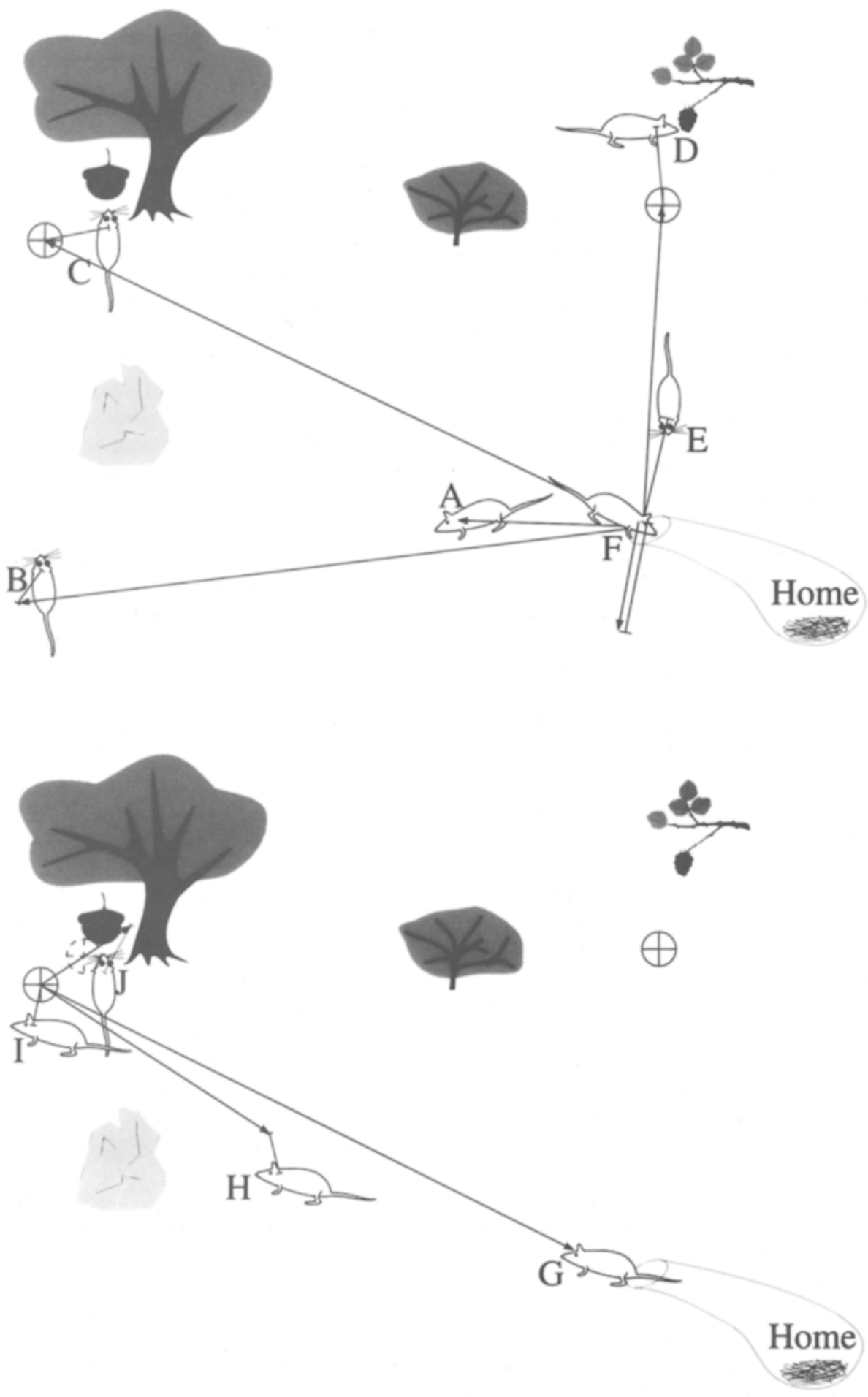

Figure 11. Single path integrators that can reset anywhere and are capable of using any coordinate as a goal. An animal goes on the same foraging trip as that in Figure 6. At C, it finds acorns under an oak tree. It stores the coordinates of its estimated position, which may not coincide with its actual position, in memory (shown here as crosshairs in a circle). Continuing on its foraging trip, it finds blackberries at $D$ and again stores the coordinates of its estimated position in memory. At $E$, the animal arrives at the estimated position of home; at $\mathrm{F}$, it is home and must reset its path integrator before its next trip. When the animal sets out to go to the oak tree (G), it uses the stored coordinates of the tree as its goal. From these coordinates and the coordinates of its own position, it derives its estimated position relative to the stored coordinates. The stored coordinates may not coincide with the actual location of the goal. While the animal is on the way to the goal, there will likely be some error in path integration (H). When the animal arrives at the estimated goal location, it may not be at the goal or even at the actual location where it last stored coordinates. It will need to search for the goal. When there $(J)$, it can average its current estimated position (in this figure, roughly northeast of its actual location) with the stored coordinates to arrive at a better estimate of the goal location. 
McNaughton et al., 1996; Redish \& Touretzky, 1997; Samsonovich \& McNaughton, 1997; Tanila, Shapiro, \& Eichenbaum, 1997; Touretzky \& Redish, 1996; Wan et al., 1994).

\section{SUMMARY AND FURTHER IMPLICATIONS}

The number of resetting points and goals a path integrator can deal with are features that are crucial for determining how useful path integration is in navigation. The results of most studies of path integration can be accounted for by use of a single path integrator keeping track of only one goal and resetting only at the goal. However, Etienne et al. (1991; Etienne et al., 1998; Etienne, Maurer, \& Séquinot, 1996) reported that hamsters can use path integration not only to return home but also to find again the location of food they had been shown. Humans can keep track of several destinations while deprived of location-based information (Farrell \& Thomson, 1993; Lee \& Thomson, 1982; Loomis et al., 1993; Rieser et al., 1986; Thomson, 1983a, 1983b). Bees also have the capacity to keep track of at least two goals at a time. Their waggle dances specify only the distance and direction of a destination, not its spatial relationship to landmarks. Bees, therefore, must use path integration to find a foraging patch specified in a waggle dance. The distance that dancing foragers communicate is itself determined through path integration, and the return flight is also controlled by path integration (Esch \& Burns, 1996; Kirchner \& Braun, 1994; Srinivasan, Zhang, Lehrer, \& Collett, 1996). Bees can store the coordinates of start point and destination and are able to switch from one destination to another (Lindauer, 1963; see the section on Multiple Independent Path Integrators, Each With Single Resetting Point and Goal). There is also evidence that bees, rats, hamsters, and humans can reset path integration at locations other than home (Gladwin, 1970; Gothard et al., 1996b; Griffin \& Etienne, 1998; Lewis, 1994; Srinivasan et al., 1997). Therefore, it appears that bees and humans have at least one path integrator that can deal with multiple goals and resetting points.

A path integrator with multiple goals and resetting points would be an ideal substrate for a cognitive map. Indeed, Gould (1986) suggested that bees do have a cognitive map, on the grounds that they were able to fly a novel shortcut between two familiar places. However, it was later found that this ability depended on the bees' being able to see landmarks visible from the destination (Dyer, 1991; Dyer, Berry, \& Richard, 1993; Wehner \& Menzel, 1990). In the absence of such landmarks, bees first returned to the hive, rather than shortcutting. How can this failure to shortcut be reconciled with my argument that bees use a path integrator that provides an ideal substrate for cognitive mapping and that should make shortcutting a trivial problem? After all, when bees were caught on their way to a feeder and displaced to a familiar place, they only needed to do three things in order to shortcut. First, they had to identify the place, which they were evidently able to do, given that they returned directly to the hive. Second, after identification of the place to which they had been carried, they should have retrieved the coordinates of this release site in order to reset. Third, the bees must retrieve as their goal coordinates those of their original destination, rather than those of the hive. The necessity of the second and third steps means that a failure of memory retrieval could account for bees' failure to shortcut even if representation and readout are adequate for the task.

A second line of reasoning leads to the same point. Bees can average two vectors (T. S. Collett, Baron, \& Sellen, 1996; Menzel et al., 1996; Menzel, Geiger, Müller, Joerges, \& Chittka, 1998), and vector averaging is vector addition and then division by the number of vectors. Why do bees not use that capacity for vector addition to create shortcuts? A factor that could account for the discrepancy is the presence or absence of visual retrieval cues. Bees failed to shortcut when they were passively transported to a place that was familiar but at which no landmarks were present that were also visible from the bees' intended destination. However, landmarks could have served not only as beacons or part of a snapshot to be used for image matching (Cartwright \& Collett, 1982, 1983), but also as retrieval cues for vectors. In both of the studies demonstrating vector averaging, the bees had learned to fly along two vectors, each associated with a different visual scene. Vector averaging occurred when the bees saw a scene intermediate to those serving as retrieval cues for the learned vectors.

That leads to two possible explanations for bees' failure to shortcut. If bees can retrieve vectors only after being cued by a visual scene, that constraint on memory retrieval, not spatial ability, would prevent them from shortcutting in experiments designed not to give such visual cues. Alternatively, visual cues might not be necessary retrieval cues but might be strong enough to override other motivations. The bees' starting point was familiar because they had repeatedly shuttled back and forth between a feeder there and the hive. The panorama surrounding this feeding place could be strongly associated with a return vector, taking priority over computing a shortcut. In this case, a different way of giving bees information regarding starting point and destination might reveal an ability to use shortcuts. Bees could be trained to a food source just rich enough to keep them foraging, but poor enough that they would switch to a richer source indicated by another forager's waggle dance. If they found no food when arriving at that new location, which is not yet strongly associated with a return vector, would they shortcut to the familiar food source?

Regardless of whether an ability to use shortcuts might be revealed by such a procedure, under most conditions, bees fail the shortcut criterion for cognitive mapping (Dyer, 1991; Dyer et al., 1993; Wehner \& Menzel, 1990). However, the point here is that there are many ways in which an animal's navigation system may fail at cognitive mapping. In the case of the bee, the shortcoming may 
be not in the representation or even the readout mechanism, but in the type of information the bee uses to cue retrieval of goal coordinates.

There is a further useful feature a path integrator might have, which is best explained by an example. Imagine I am sailing in fog on a large but shallow lake, navigating by path integration. I know from experience that my navigational error is in the range of $10 \%$. After sailing for $10 \mathrm{~km}$, I encounter a wreck where none should be, within the limits of my uncertainty. I mark it on my chart as a hazard to shipping, with a $1-\mathrm{km}$ circle around that position representing my confidence interval. After sailing on for another $2 \mathrm{~km}$, the fog lifts, and I can fix my position. I find that I am $600 \mathrm{~m}$ south of my estimated position. That allows me to update retrospectively the estimated position of the wreck to $600 \mathrm{~m}$ further south, with a confidence interval now of $200 \mathrm{~m}$, which represents the uncertainty of my navigation between the wreck and my position fix. Note that both position and uncertainty are updated.

Although the example shows that retrospective updating is part of formal navigation methods, I am not aware of any convincing evidence that it occurs under more naturalistic conditions, even in humans. When bees and ants repeatedly experience conflicting motion-based information on the outward and return journey of a displacement experiment, they compromise between point of arrival and point of departure (M. Collett et al., 1999; Otto, 1959). The compromise could be achieved by retrospective updating of coordinates on arrival at the hive or nest, but M. Collett et al. suggested an alternative interpretation. The issue remains open for the moment.

\section{CONCLUSIONS}

Several authors have suggested that path integration could be the basis of cognitive mapping (Gallistel, 1990; McNaughton et al., 1996; O'Keefe, 1976; Redish \& Touretzky, 1997; Samsonovich \& McNaughton, 1997; Touretzky \& Redish, 1996; Wan et al., 1994), rather than a simpler alternative that must be ruled out (Bennett, 1996). I suggest that it depends on what type of path integrator a species uses. At one extreme, a path integrator with a single goal and resetting point clearly cannot be the basis for cognitive mapping. It represents only the spatial relationship between the current position and one fixed goal, providing nothing more than a lifeline that always tells an animal where home is. At the other extreme, path integrators capable of dealing with multiple goals provide the unified, internally consistent, metric representation with efficient information storage that O'Keefe and Nadel (1978) considered characteristic of cognitive mapping. They would enable an animal to plan a path to any point with known coordinates, including novel shortcuts (given good enough readout and retrieval mechanisms). This would be possible by doing nothing more sophisticated with landmarks than using them to recognize resetting points-for example, by matching the cur- rent visual scene to a stored retinal snapshot. A sufficient density of resetting points can keep the accumulation of random errors within any desired bounds, and the accuracy of resetting and goal coordinates can be ensured through an averaging process. Path integration can be the basis of sophisticated and flexible navigation.

\section{REFERENCES}

Benhamou, S. (1997a). On systems of reference involved in spatial memory. Behavioural Processes, 40, 149-163.

Benhamou. S. (1997b). Path integration by swimming rats. Animal Behaviour, 54, $321-327$.

Benhamou, S., \& Bovet, P. (1992). Distinguishing between elementary orientation mechanisms by means of path analysis. Animal Behaviour, 43, 371-377.

Benhamou, S., Sauvé, J. P., \& Bovet, P. (1990). Spatial memory in large-scale movements: Efficiency and limitation of the egocentric coding process. Journal of Theoretical Biology, 145, 1-12.

BENNETT, A. T. D. (1996). Do animals have cognitive maps? Journal of Experimental Biology, 199, 219-224.

Beugnon, G., \& Campan, R. (1989). Homing in the field cricket, GrylIus campestris. Journal of Insect Behaviour, 2, 187-198.

Biegler, R., \& MorRIS, R. G. M. (1996). Landmark stability: Further studies pointing to a role in spatial learning. Quarterly Journal of Experimental Psychology, 49B, 307-345.

Bureš, J., Burešová, O., \& Nerad, L. (1992). Can rats solve a simple version of the traveling salesman problem? Behavioural Brain Research, 52, 133-142.

BURGESS, N., \& O'KEEFE, J. (1995). Modelling spatial navigation by the rat hippocampus. International Journal of Neural Systems, 6(Suppl.), 87-94.

Burgess, N., Recce, M., \& O'KeEFe, J. (1994). A model of hippocampal function. Neural Networks, 7, 1065-1081.

Cartwright, B. A., \& Collett, T. S. (1982). How honey bees use landmarks to guide their return to food. Nature, 295, 560-564.

Cartwright, B. A., \& ColletT, T. S. (1983). Landmark learning in bees: Experiments and models. Journal of Comparative Physiology, 151, 521-543.

ChenG, K. (1990). More psychophysics of the pigeon's use of landmarks. Journal of Comparative Physiology A, 166, 857-863.

Cheng, K., Srinivasan, M. V., \& Zhang, S. W. (1999). Error is proportional to distance measured by honeybees: Weber's law in the odometer. Animal Cognition, 2, 11-16.

ChittKa, L., \& Kunze, J. (1995). The significance of landmarks for path integration in homing honeybee foragers. Naturwissenschaften, 82, 341-343.

Collett, M., Collett, T. S., \& Wehner, R. (1999). Calibration of vector navigation in desert ants. Current Biology, 9, 1031-1034.

Collett, T. S., Baron, J., \& Sellen, K. (1996). On the encoding of movement vectors by honeybees: Are distance and direction represented independently? Journal of Comparative Physiology A, 179, 395-406.

Cramer, A. E., \& Gallistel, C. R. (1997). Vervet monkeys as travelling salesmen. Nature, 387, 464.

DAVIS, E. (1986). Representing and acquiring spatial knowledge. London: Pitman.

DYER, F. C. (1991). Bees acquire route-based memories but not cognitive maps in a familiar landscape. Animal Behaviour, 41, 239-246.

Dyer, F. C., Berry, N. A., \& Richard, A. S. (1993). Honey bee spatial memory: Use of route-based memories after displacement. Animal Behaviour, 45, 1028-1030.

EASTON, R. D., \& ShOLl. M. J. (1995). Object-array structure, frames of reference, and retrieval of spatial knowledge. Journal of Experimental Psychology: Learning, Memory, \& Cognition, 21, 483-500.

ЕsCh, H. E., \& BurNS, J. E. (1996). Distance estimation by foraging honeybees. Journal of Experimental Biology, 199, 155-162.

Etienne, A. S., Hurni, C., Maurer, R., \& Séguinot, V. (1991). Twofold path integration during hoarding in the golden hamster? Ethology, Ecology \& Evolution, 3, 1-11. 
Etienne, A. S., Maurer, R., Berlie, J., Reverdin, B., Rowe, T., Georgakopoulos, J., \& SÉGUiNot, V. (1998). Navigation through vector addition. Nature, 396, 161-164.

Etienne, A. S., Maurer, R., \& SAUCy, F. (1988). Limitations in the assessment of path dependent information. Behaviour, 106, 81-111.

Etienne, A. S., Maurer, R., Saucy, F., \& Teroni, E. (1986). Shortdistance homing in the golden hamster after a passive outward journey. Animal Behaviour, 34, 696-715.

Etienne, A. S., Maurer, R., \& Séguinot, V. (1996). Path integration in mammals and its interaction with visual landmarks. Journal of Experimental Biology, 199, 201-209.

Etienne, A. S., Teroni, E., Hurni, C., \& Portenier, V. (1990). The effect of a single light cue on homing behaviour of the golden hamster. Animal Behaviour, 39, 17-41.

Etienne, A. S., Teroni, E., Maurer, R., Portenier, V., \& Saucy, F. (1985). Short distance homing in a small mammal: The role of exteroceptive cues and path integration. Experientia, 41, 122-125.

FarRell, M. J., \& Thomson, J. A. (1993). Direct and computational mechanisms in spatial updating. In S. S. Valenti \& J. B. Pittenger (Eds.), Studies in perception and action $I I$ (pp. 172-175). Hillsdale, NJ: Erlbaum.

FarRell, M. J., \& ThOmson, J. A. (1998). Automatic spatial updating during locomotion without vision. Quarterly Journal of Experimental Psychology, 51 A, 637-654.

GaLLISTEL, C. R. (1990). The organization of learning. Cambridge, MA: MIT Press, Bradford Books.

Gallistel, C. R., \& Cramer, A. E. (1996). Computations on metric maps in mammals: Getting oriented and choosing a multi-destination route. Journal of Experimental Biology, 199, 211-217.

Gladwin, T. (1970). East is a big bird: Navigation and logic on Puluwat atoll. Cambridge, MA: Harvard University Press.

Gothard, K. M., Skaggs, W. E., \& McNaughton, B. L. (1996a). Binding of hippocampal CAl neural activity to multiple reference frames in a landmark-based navigation task. Journal of Neuroscience, 16, 823-835.

Gothard, K. M., Skaggs, W. E., \& McNaughton, B. L. (1996b). Dynamics of mismatch correction in the hippocampal ensemble code for space: Interaction between path integration and environmental cues. Journal of Neuroscience, 16, 8027-8040.

GouLD, J. L. (1986). The locale map of honey bees: Do insects have cognitive maps? Science, 232, 861-863.

GrifFIN, A. S., \& ETIENNE, A. S. (1998). Updating the path integrator through a visual fix. Psychobiology, 26, 240-248.

HARTMANN, G., \& WeHNER, R. (1995). The ant's path integration system: A neural architecture. Biological Cybernetics, 73, 483-497.

HoffMAnN, G. (1984). Orientation behaviour of the desert woodlouse Hemilepistus reaumuri: Adaptations to ecological and physiological problems. Symposium of the Zoological Society of London, 53, 405-422.

JANDER, R. (1957). Die optische Richtungorientierung der roten Waldameise (Formic rufa L.) [The optical directional orientation of the red forest ant (Formica rufa L.). Zeitschrift für vergleichende Physiologie, 40, 162-238.

JefFERY, K. J., DONNETT, J. G., BuRgess, N., \& O'KeEFe, J. (1997). Directional control of hippocampal place cells. Experimental Brain Research, 117, 131-142.

Jung, M. W., Wiener, S. I., \& McNaughton, B. L. (1994). Comparison of spatial firing characteristics of units in dorsal and ventral hippocampus of the rat. Journal of Neuroscience, 14, 7347-7356.

KamIL, A. C., \& JoNES, J. J. (1997). The seed-storing corvid Clark's nutcracker learns geometric relationships among landmarks. Nature, $390,276-279$

KEITH, J. R., \& MCVETY, K. M. (1988). Latent place learning in a novel environment and the influences of prior training in rats. Psychobiology, 16, 146-151.

KirChNER, W. R., \& Braun, U. (1994). Dancing honey bess indicate the location of food sources using path integration rather than cognitive maps. Animal Behaviour, 48, 1437-1441.

LEE, D. N., \& Thomson, J. A. (1982). Vision in action: The control of locomotion. In D. J. Ingle, M. A. Goodale, \& R. J. W. Mansfield (Eds.),
Analysis of visual behavior (pp. 411-433). Cambridge, MA: MIT Press.

LEwIS, D. (1994). We, the navigators. Honolulu: University of Hawaii Press.

LINDAUER, M. (1963). Kompaßorientierung [Compass orientation]. Ergebnisse der Biologie, 26, 158-181.

LoOmis, J. M., Klatzky, R. L., Golledge, R. G., Cicinelli, J. G., PelleGRINO, J. W., \& FRY, P. A. (1993). Nonvisual navigation by blind and sighted: Assessment of path integration ability. Journal of Experimental Psychology: General, 122, 73-91.

MAURER, R. (1998). A connectionist model of path integration with and without a representation of distance to the starting point. Psychobiology, 26, 21 -35.

MAURER, R., \& SÉguinot, V. (1995). What is modelling for? A critical review of the models of path integration. Journal of Theoretical Biology, 175, 457-475.

McNaughton, B. L, Barnes, C. A., Gerrard, J. L., Gothard, K. M., Jung, M. W., Knierim, J. J., Kudrimoti, H., QIn, Y., Skaggs, W. E., SUSTER, M., \& WEAVER, K. L. (1996). Deciphering the hippocampal polyglot: The hippocampus as a path integration system. Journal of Experimental Biology, 199, 165-171.

Menzel, R., Geiger, K., Chittka, L., Joerges, J., Kunze, J., \& MüLLER, U. (1996). The knowledge base of bee navigation. Journal of Experimental Biology, 199, 141-146.

Menzel, R., Geiger, K., Müller, U., Joerges, J., \& Chittka, L. (1998). Bees travel novel homeward routes by integrating separately acquired vector memories. Animal Behaviour, 55, 139-152.

Mittelstaedt, H. (1983). The role of multimodal convergence in homing by path integration. Fortschritte der Zoologie, 28, 197-212.

MittelstaedT, H. (1985). Analytical cybernetics of spider navigation. In F. G. Barth (Ed.), Neurobiology of arachnids (pp. 298-316). Berlin: Springer-Verlag.

Mittelstaedt, M. L., \& Glasauer, S. (1991). Idiothetic navigation in gerbils and humans. Zoologisches Jahrbuch: Physiologie, 95, 427. 435.

MittelstaedT, H., \& Mittelstaedt, M. L. (1973). Mechanismen der Orientierung ohne richtende Außenreize [Mechanisms of orientation without guiding external cues]. Fortschritte der Zoologie. 21, 46-58.

Müller, M., \& WeHNER, R. (1988). Path integration in desert ants, Cataglyphis fortis. Proceedings of the National Academv of Sciences, $85,5287-5290$.

O'KeEFE, J. (1976). Place units in the hippocampus of the freely moving rat. Experimental Neurology, 51, 78-109.

O'KeEFE, J., \& NADEL, L. (1978). The hippocampus as a cognitive map. Oxford: Oxford University Press, Clarendon Press.

Oтто, F. (1959). Die Bedeutung des Rückfluges für die Richtungs- und Entfernungangabe der Bienen [The significance of the return flight for the indication of direction and distance in bees]. Zeitschrift für Vergleichende Physiologie, 15, 303-333.

PoucET, B. (1993). Spatial cognitive maps in animals: New hypotheses on their structure and neural mechanisms. Psychological Review, 100, 163-182.

REDish, A. D., \& TouretzKy, D. S. (1997). Cognitive maps beyond the hippocampus. Hippocampus, 7, 15-35.

Reid, A. K., \& StadDON, J. E. R. (1998). A dynamic reader for the cognitive map. Psychological Review 4 105, 585-601.

RIESER, J. J., GUTH, D. A., \& EvereTt, E. W. (1986). Sensitivity to perspective structure while walking without vision. Perception, 15 , 173-188.

Ronacher, B., \& WeHner, R. (1995). Desert ants Cataglyphis fortis use self-induced optic flow to measure distances traveled. Journal of Comparative Physiology A, 177, 21-27.

Samsonovich, A.. \& McNaughton, B. L. (1997). Path integration and cognitive mapping in a continuous attractor neuronal network model. Journal of Neuroscience, 17, 5900-5920.

SChmidt, I., Collett, T. S., Dillier, F. X., \& Wehner, R. (1992). How desert ants cope with enforced detours on their way home. Journal of Comparative Physiology A, 171, 285-288.

Séguinot, V., Cattet, J., \& Benhamou, S. (1998). Path integration in dogs. Animal Behaviour, 55, 787-797. 
SÉguinot, V., Maurer, R., \& Etienne, A. S. (1993). Dead reckoning in a small mammal: The evaluation of distance. Journal of Compar. ative Physiology $A, 173,103-113$.

Srinivasan, M. V., Zhang, S. W., \& BidWell, N. (1997). Visually mediated odometry in honeybees. Journal of Experimental Biology, 200, 2513-2522.

Srinivasan, M. V., Zhang, S. W., Lehrer, M., \& Collett, T. S. (1996). Honeybee navigation en route to the goal: Visual flight control and odometry. Journal of Experimental Biology, 199, 237-244.

STEElE, R. J., \& MorrIS, R. G. M. (1999). Delay-dependent impairment of a hippocampal-dependent matching-to-sample task with chronic and intrahippocampal infusion of the NMDA antagonist DAP5. Hippocampus, 9, 118-136.

Tanila, H., Shapiro, M. L.. \& Eichenbaum, H. (1997). Discordance of spatial representation in ensembles of hippocampal place cells. Hippocampus, 7, 613-623.

ThOmson, J. A. (1983a). How do we use visual information to control locomotion? Trends in Neurosciences, 3, 247-250.

Thomson, J. A. (1983b), Is continuous visual monitoring necessary in visually guided locomotion? Journal of Experimental Psvchologv: Human Perception \& Performance, 9, 427-443.

TourerZKY, D. S., \& ReDISH, A. D. (1996). Theory of rodent navigation based on interacting representations of space. Hippocampus, 6 , 247-270.

voN FRISCH, K. (1967). The dance language and orientation of hees. Cambridge, MA: Harvard University Press, Belknap Press.

voN SAINT PAUL, U. (1982). Do geese use path integration for walking home? In F. Papi \& H. G. Wallraff (Eds.), Avian navigation (pp. 296307). Berlin: Springer-Verlag.
WAN, H. S., Touretzky, D. S., \& Redish, A. D. (1994). Towards a computational theory of rat navigation. In M. Mozer, P. Smolensky, D. Touretzky, J. Elman, \& A. Weigend (Eds.), Proceedings of the 1993 Connectionist Models Summer School (pp. 11-19). Hillsdale, NJ: Erlbaum.

WeHNer, R., \& MEnzel, R. (1990). Do insects have cognitive maps? Annual Review of Neuroscience, 13, 403-414.

WeHNER, R., Michel, B., \& ANtonsen, P. (1996). Visual navigation in insects: Coupling of egocentric and geocentric information. Journal of Experimental Biology, 199, 129-140.

WEHNER, R., \& RÄBER, F. (1979). Visual spatial memory in desert ants, Cataglyphis bicolor, Experientia, 35, 1569-1571.

WeHNER, R., \& SRINIVASAN, M. V. (1981). Searching behaviour of desert ants, genus Cataglyphis (Formicidae, Hymenoptera). Journal of Comparative Physiology, 142, 315-338.

WEHNER, R., \& WEHNER, S. (1990). Insect navigation: Use of maps or Ariadne's thread? Ethology, Ecology \& Evolution, 2, 27-48.

Whishaw, I. Q. (1991). Latent leaning in a swimming pool place task by rats: Evidence for the use of associative and not cognitive mapping processes. Quarterly.Journal of Experimental Psychology, 43B, 83-103.

WORDEN, R. (1992). Navigation by fragment fitting: A theory of hippocampal function. Hippocampus, 2, 165-187.

ZEIL, J. (1998). Homing in fiddler crabs (Uca lactea annulipes and Uca vomeris). Journal of Comparative Physiology A, 183, 367-377.

Zhang. K., \& Sejnowski, T. J. (1999). Neuronal tuning: To sharpen or to broaden? Neural Computation, 11, 75-84.

(Manuscript received August 24, 1999;

revision accepted for publication November 15, 1999.) 